\title{
The choice of the concept of magnetic field lines or of electric current lines: Alfvén medal lecture
}

\author{
S.-I. Akasofu \\ International Arctic Research Center, 930 Koyukuk Drive, University of Alaska Fairbanks, Fairbanks, AK 99775-7340, USA
}

Received: 3 May 2011 - Accepted: 20 June 2011 - Published: 4 July 2011

\begin{abstract}
In 1967, at the Birkeland Symposium in Sandefjord, Norway, Professor Hannes Alfvén stated that the second approach (in solving unsolved problems by the standard MHD theory) to cosmic electrodynamics is to "thaw" the "frozen-in" magnetic field lines. "We can illustrate essential properties of the electromagnetic state of space either by depicting the magnetic field lines or by depicting electric current lines," he said. There has been much progress in space physics since the Birkeland Symposium more than 40 years ago, but unfortunately our scientific community has not really succeeded in thawing the frozen-in field lines. Instead, it has pursued magnetic reconnection, a concept that Alfvén had been critical of. It is shown here that we have to study many unsolved problems and problems thought to be solved in terms of both the magnetic field line concept and the current system concept. In taking Alfvén's approach, we must consider the whole system, including the power supply (dynamo process) and its transmission and distribution (electric currents) and observed phenomena (power dissipation processes). Such a consideration can provide physical insight into many of our unsolved problems and problems thought to be solved. In this paper, we consider substorm onset processes, the substorm current system, sunspots, solar flares, coronal mass ejections, the interplanetary current sheet, and the magnetic field configuration of the heliosphere in terms of the current system concept. In particular, it is shown that a study of the current system is essential in substorm studies, more than changes of the magnetic field configuration in the magnetotail.
\end{abstract}

Keywords. Interplanetary physics (Flare and stream dynamics) - Magnetospheric physics (Solar wind-magnetosphere interactions; Storms and substorms)

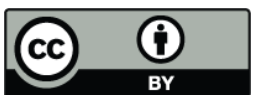

Correspondence to: S.-I. Akasofu (sakasofu@iarc.uaf.edu)

\section{Introduction}

In magnetospheric physics and solar physics, we deal mainly with electromagnetic processes. Many of the observed phenomena are manifestations of electromagnetic energy dissipation processes. Thus it is necessary to consider the power supply processes. However, when we take the magnetic field line approach, we do not necessarily consider explicitly the chain of processes consisting of dynamo processes, which supply the necessary power, electric currents, which transmit and distribute the power, and observed phenomena as resulting power dissipation processes. Instead, we have considered those problems only in terms of moving magnetic field lines. The concept of magnetic field lines is useful, but moving magnetic field lines are related to changing electric currents. We have to understand the physics involved in changing electric currents.

Magnetic fields other than those of permanent magnets must be produced by electric currents, which are produced by particle motions; changing magnetic fields are caused by changing electric currents. What Alfvén emphasized in reviewing the progress in cosmic electrodynamics was that even if current $\boldsymbol{i}$ is difficult to measure, and thus should be determined by curl $\boldsymbol{B}$, the whole current system, including its generation, the transmission, and dissipation, should be considered together. In fact, we deal with many current systems - ionospheric currents, field-aligned currents, the ring current, the Chapman-Ferraro current, the cross-tail current, the solar equatorial current sheet, the force-free field current $(\boldsymbol{i} \times \boldsymbol{B})$ for solar flares and others.

The success of the magnetic reconnection theory proposed by Dungey (1961) has become the prevailing view in magnetospheric physics, so that we tend to look at solar and magnetospheric phenomena through a filter that can recognize only magnetic field lines and their movements and try to understand magnetic reconnection as the only process for dissipating magnetic energy. In a scientific field, it is

Published by Copernicus Publications on behalf of the European Geosciences Union. 


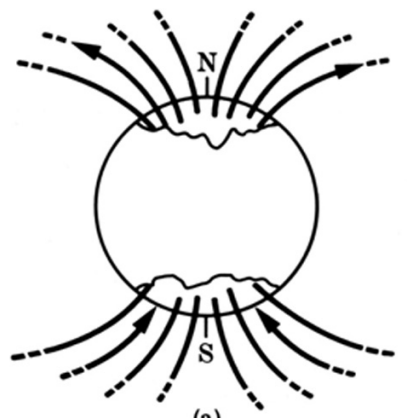

(a)

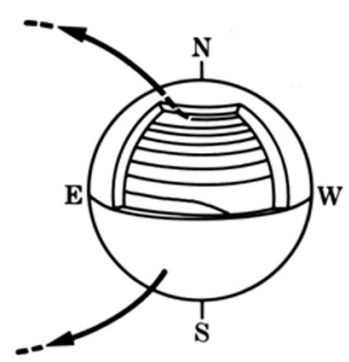

(c)

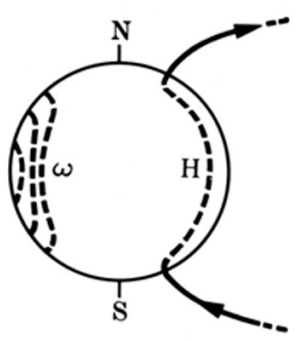

(b)

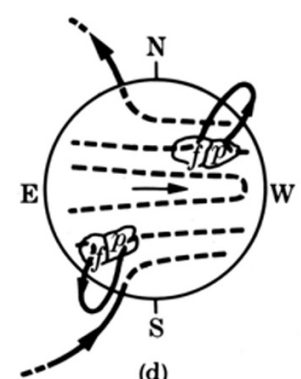

(d)
Fig. 1a. Schematic illustration of how sunspot pairs are formed by the non-uniform rotation of the Sun (Babcock, 1961).

dangerous to rely on only one concept. Alfvén's point was that we should also use a filter that can see electric currents (Alfvén, 1967, 1977, 1981, 1986).

For these reasons, this paper takes an "unconventional" approach, namely, Alfvén's approach based on electric currents. In doing so, however, a somewhat different approach from Alfvén's is taken; Alfvén based his conclusions on laboratory experiments, while this paper is based on observed facts. These observed facts have often been overlooked, ignored, or discredited because they do not fit in the prevailing theories. Although this approach may appear as a backward step from the point of view of the developed magnetic reconnection paradigm, those observed facts are neither trivial nor exceptional nor abnormal.

Alfvén (1951) and Chapman (1951) had a long debate, but actually both were correct in the sense that a pencil has two ends. Chapman emphasized one end, the plasma aspects of the solar stream, while Alfvén stressed the other end, the importance of the interplanetary magnetic field. Dungey (1961) combined both, and thus we have the present concept of the magnetosphere. However, both magnetic field line and current line approaches are needed to advance one step beyond Dungey's concept.

It is hoped that the discussion based on the observed facts and electric currents will be useful in pointing out a different view and in understanding unsolved problems and problems thought to be understood.
(1) HYPOTHETICAL SUBMERGED FLUX TUBE

(2)

LIFTING

MECHANISM

\section{(3)} ANNIHILATION
MECHANISM
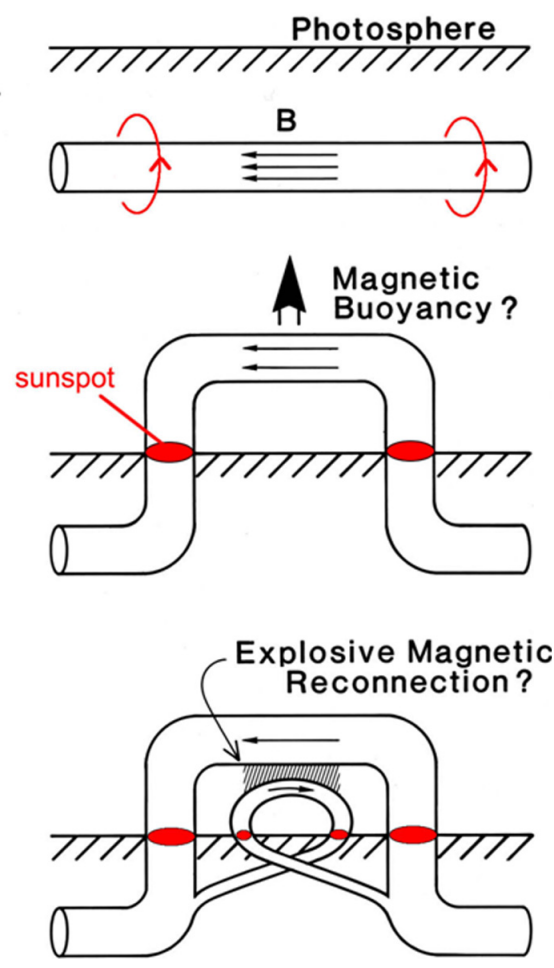

Fig. 1b. Schematic illustration of how a tube of magnetic flux forms a sunspot pair, and how a solar flare is supposed to occur by magnetic reconnection. In the top diagram, the red circles represent the solenoidal current.

\section{Sunspots}

Several sunspot observations may provide the simplest example of why the accepted view of sunspots fails to explain several crucial features of sunspots.

Generations of students have been taught that the nonuniform rotation of the Sun winds up a dipolar field lines, producing a tube of azimuthal magnetic field flux beneath the photosphere. As the flux in the tube increases above some critical level $\left(B^{2} / 8 \pi>p\right)$ by squeezing plasma out, the tube breaks through the photosphere by magnetic buoyancy. Two cross-sections of the tube are said to be identified as a sunspot pair (Babcock, 1961; Figs. 1a and b).

Besides many unsolved problems about sunspots, such as the 11-year sunspot cycle and the butterfly diagram, one might ask two simple or even naïve questions.

1. How is the solenoidal electric current formed in order to produce a tube of magnetic field line flux? (See Fig. 1b.)

2. How can one explain single (or isolated) spots that are of common appearance? Those spots are of common occurrence, not exceptional (see Fig. 2.)

These questions have so far not been addressed. On the other hand, a few observational facts have been overlooked or ignored. 


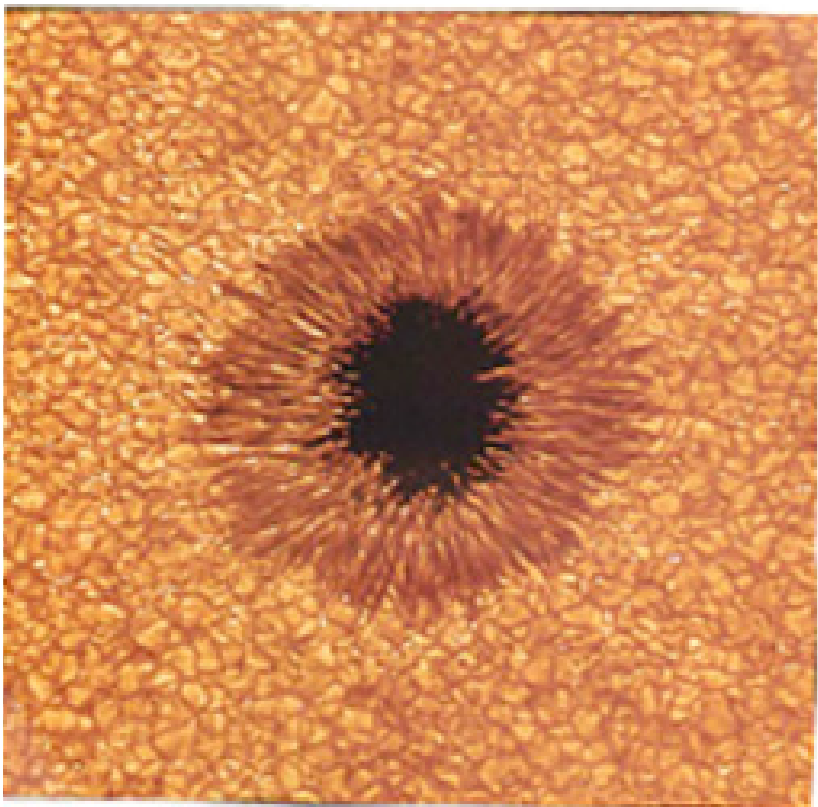

Fig. 2. An example of a single sunspot (courtesy of the Big Bear Solar Observatory).

1. There are areas of unipolar (positive or negative) regions of weak fields (UM-regions) that are distributed fairly systematically on the photosphere (Babcock and Babcock, 1955). They are the remains of old sunspots (Fig. 3).

2. A positive spot is formed in a positive UM-region, and a negative spot in a negative UM-region (see Fig. 3).

3. A sunspot pair is formed where positive and negative UM-regions are located side by side (Fig. 3).

These observational facts are difficult to explain in terms of a rising magnetic tube of force. This is because the rising magnetic flux tubes (sunspot pairs) are supposed to occur randomly, irrespective of the location of the sign of UM-regions.

After all, the presence of a magnetic tube of force beneath the photosphere is simply a long-held hypothesis; there has been no observational evidence. Thus one might consider other possibilities based on observations, rather than the hypothesis.

Single spots provide a hard problem to explain in terms of the magnetic flux hypothesis. Based on the observed fact (2), one obvious possibility for the formation of a single sunspot is the convergence of a part of the UM-region within a small circular region. A positive circular spot requires a counterclockwise current in the photosphere. This can be generated by a radial convergence of the photospheric gas at a speed of about $100 \mathrm{~m} \mathrm{~s}^{-1}$ in a positive UM-region (say, 10 Gauss) from an area of $1000 \mathrm{~km}$ radius, namely, a dynamo process $\boldsymbol{V} \times \boldsymbol{B}$ (Akasofu, 1984) (see Fig. 4a). It should be noted

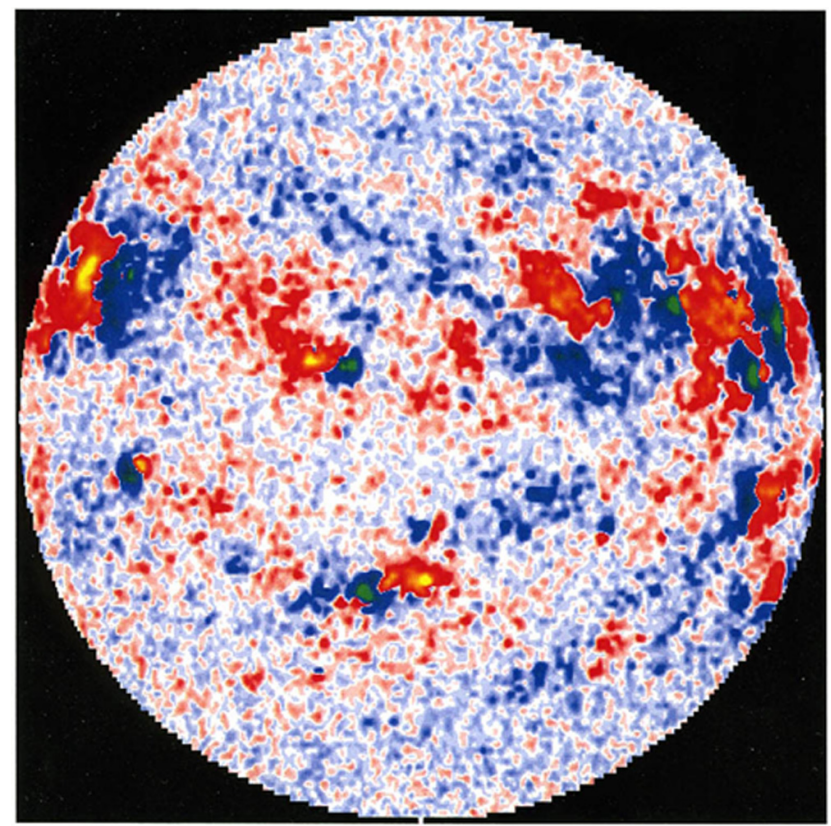

http://www.astro.ucla.edu/ obs/cur_mag_felb.png

$6 / 24 / 2001$

Fig. 3. An example of a solar magnetic observation. Yellow spots are positive sunspots located in positive UM-regions, and green spots are negative spots located in negative UM-regions (courtesy of the Kitt Peak Solar Observatory).

that the ionization rate of the photosphere is similar to that of the ionosphere $\left(10^{-6}\right)$. The conductivity is similar to that of sea water (Cowling, 1953), although the relative velocity between the neutral component and the ionized component should be examined (Akasofu and Chapman, 1972; Sect. 4.4).

It has long been known that there is a strong divergent flow of plasma called the "Evershed flow" from the upper part of a spot (see Fig. 4b). This must be caused by the needed converging flow in the lower part of a spot, because the divergent flow provides evidence that plasma is squeezed out by the radial converging flow in a spot. It is known that the penumbra of active sunspots tend to show a vortex structure; it is expected that the coriolis force can affect the radial flow as the sun is rotating. In some cases, the whole sunspot shows a vortex structure (Fig. 4b), although it is not to advocate a cyclone-like process in this paper.

The formation of a sunspot pair may be explained in the following way on the basis of the observed fact (3). A negative sunspot can be formed as a counterpart of a positive spot when a positive spot is formed near an adjacent negative UM-region, because a positive and the adjacent negative UM-regions are connected by the magnetic field lines. The converging flow in the positive UM-region induces a converging flow at the conjugating area in the negative UMregion, inducing a clockwise circular current. This process is 

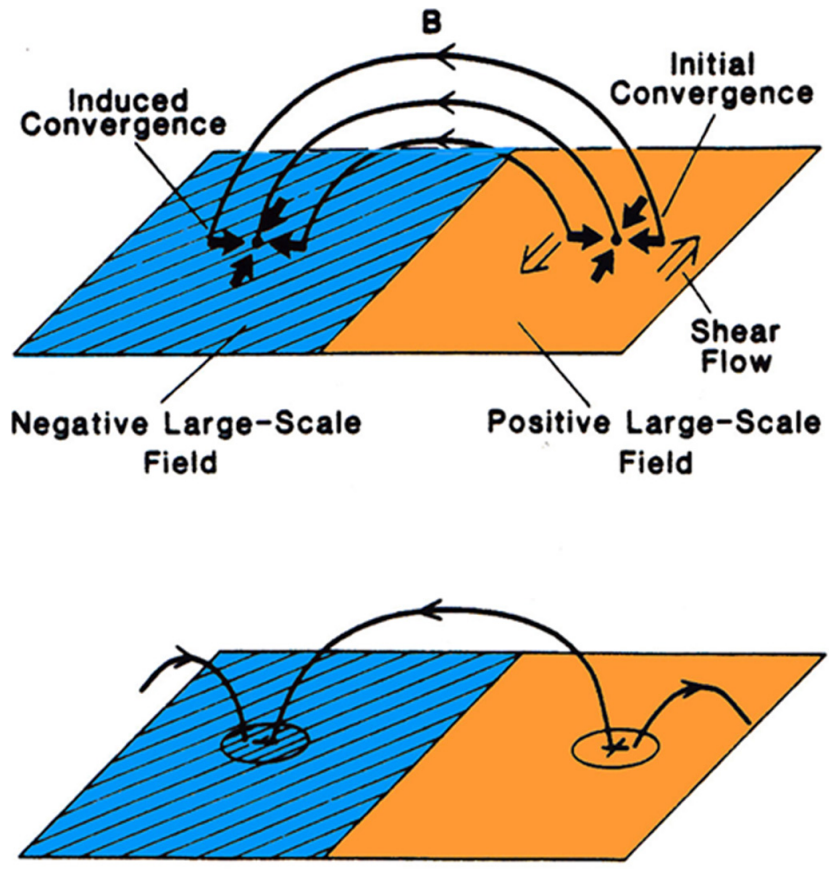

SUNSPOT PAIR FORMATION

Fig. 4a. Schematic illustration of how a sunspot pair can be formed across two adjacent UM-regions of positive and negative polarities.
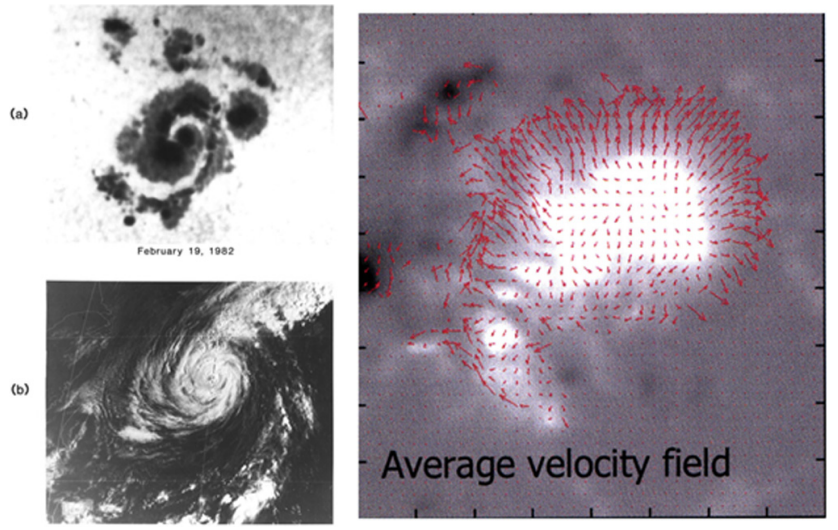

Fig. 4b. Upper left: a sunspot with a vortex structure; lower left, a typhoon near Japan; right: the Evershed flow.

illustrated in Fig. 4a. The reversal of the polarity of a sunspot pair in the other hemisphere can be explained by the fact that the polarity of the UM-regions is reversed in the other hemisphere. Figure 5 shows schematically the two views of sunspots, one through the magnetic field line filter and the other through the current line filter.

The above consideration is based on the observations, not a hypothesis, and it can further explain a few more observed facts than the submerged tube hypothesis does. When a new idea can explain a few more observations than an old one, it

\section{SUNSPOTS}

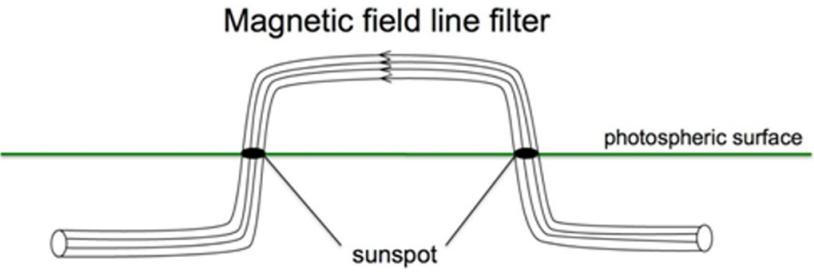

Electric current filter

photospheric surface
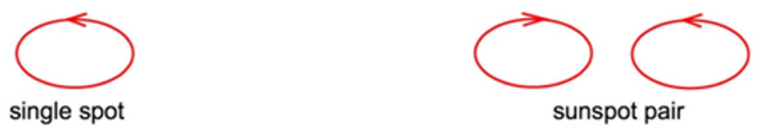

Fig. 5. The two views (magnetic field lines or electric currents) of sunspots.

may be adopted at least as an additional or alternative possibility on the basis of scientific practice, even though it may eventually be proved to be incorrect. The idea proposed here cannot explain many other aspects of sunspots, such as the occurrence of the primary spot. Nevertheless, this step may be one way by which science can make a little progress.

Some observations show that magnetic field lines in a complex sunspot group connect and disconnect "much as they do in a vacuum" in a highly conductive solar corona (Sheeley et al., 1975), although such a potential consideration is only a rough first approximation. Figure 6a shows that the field lines from a sunspot pair are not necessarily connected within the pair, and the field lines from adjacent sunspot pairs are connected just as in a vacuum. In fact, some field lines of two sunspot groups in the two hemispheres are often connected across the equator. Therefore, the magnetic tube hypothesis shown in Fig. $6 \mathrm{~b}$ does not seem to work.

Further, Sheeley et al. (1975) observed that magnetic field lines connect or disconnect just as in a vacuum in a highly conductive corona when a new sunspot pair appears without solar flares (Sect. 5). Such magnetic changes must be caused by changes of currents in the photosphere.

Even if the above considerations of sunspots may be unconventional, a solenoidal current is absolutely needed in forming a magnetic tube of force. The question is where and how it can be formed, if it would exist.

\section{Solar wind-magnetosphere dynamo}

The solar wind-magnetosphere dynamo along the magnetopause results from the fact that the solar wind blows through the IMF field lines connected with the magnetospheric field lines (Fig. 7a). The dynamo process on 


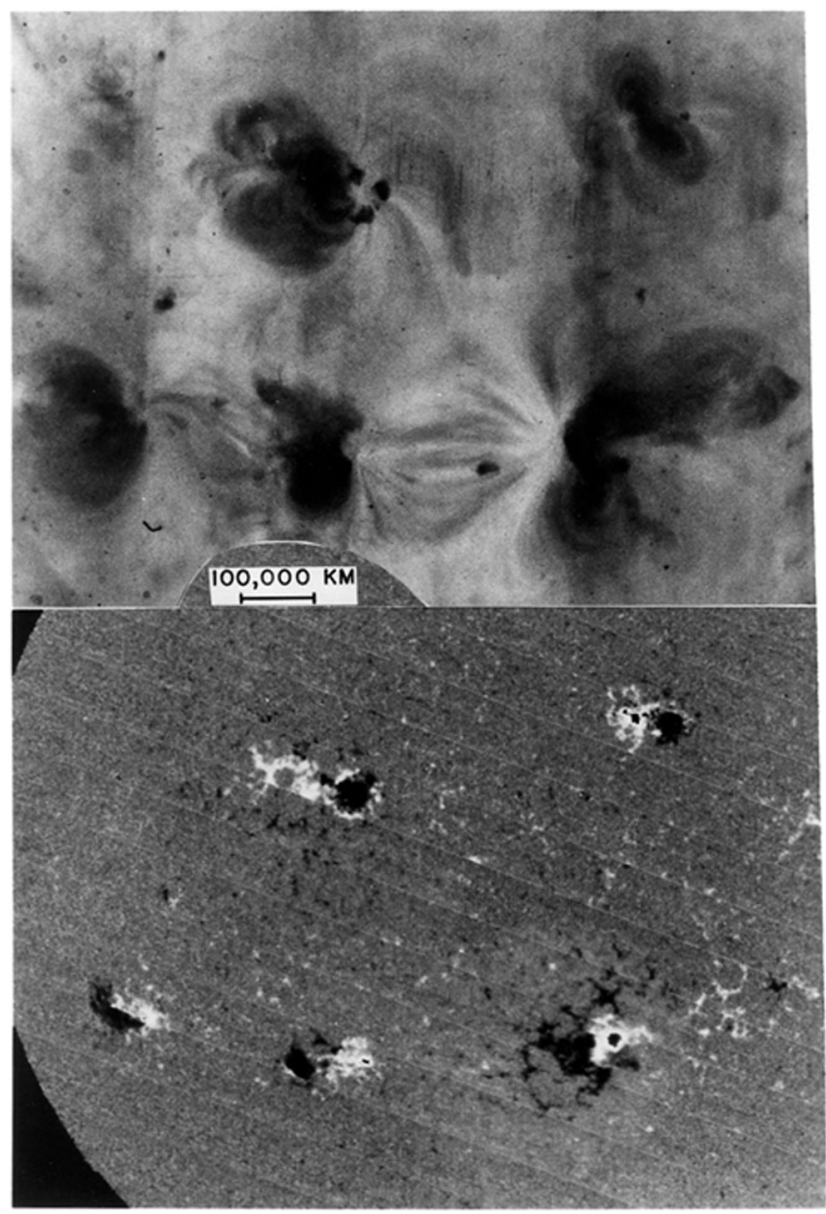

Fig. 6a. The magnetic field lines among five sunspot pairs (two in the Northern Hemisphere and three in the Southern Hemisphere) are connected almost as in a vacuum, in spite of the fact that they are embedded in a highly conductive plasma (Sheeley et al., 1975).

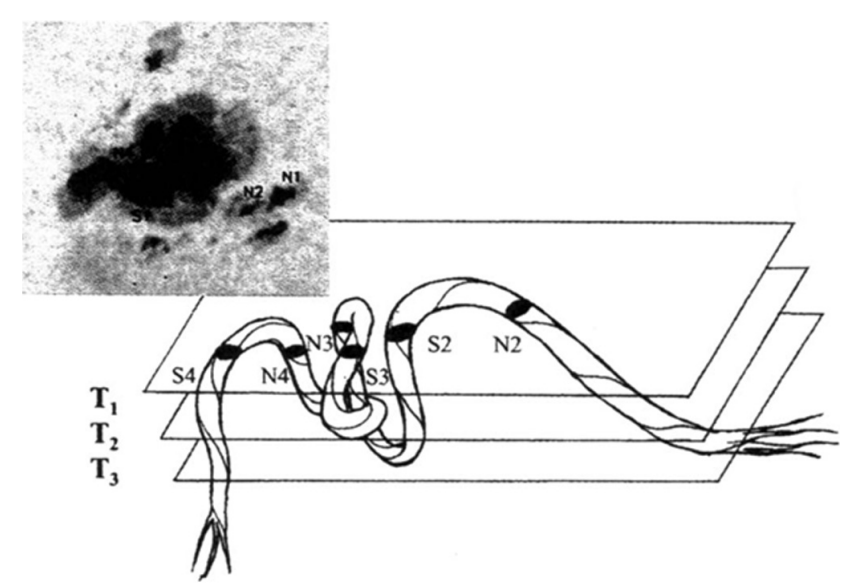

Fig. 6b. Attempt to explain the structure of a sunspot group on the basis of a flux tube concept (Deng et al., 2005).

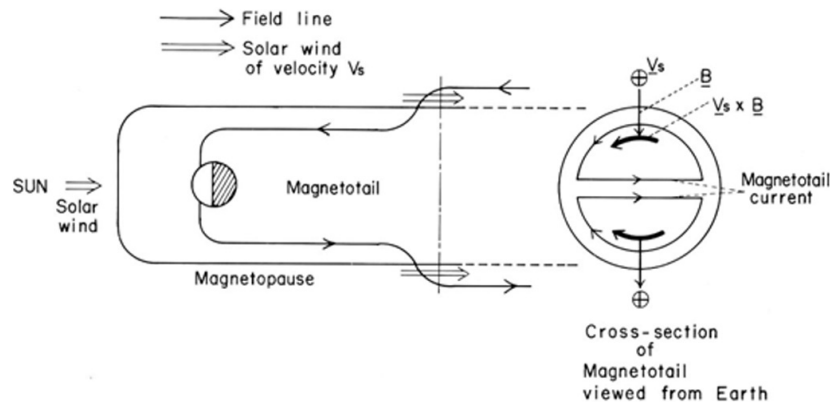

Fig. 7a. Schematic illustration of how the solar windmagnetosphere dynamo works.

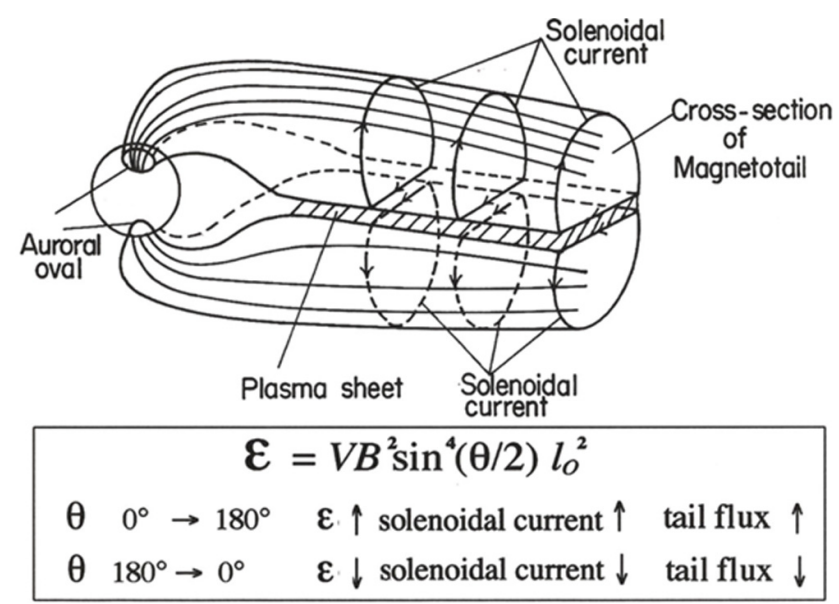

Fig. 7b. The two semicircular solenoidal currents in the magnetotail. The flux change in the magnetotail is associated with changing power $\varepsilon$.

the magnetopause $( \pm e \boldsymbol{V} \times \boldsymbol{B})$ produces two semi-circular solenoidal currents in the magnetotail (Fig. 7a); both northern and southern semi-circular currents join along the plasma sheet, constituting the cross-tail current (Fig. 7b); see also Akasofu et al. (1981). The two solenoidal currents cause apparent "stretching" of the dipole field lines. The two solenoidal currents have a finite length, so that the magnetic field lines produced by the two currents are connected to each other near both the front end and the tailward end. Figure 8 shows schematically this situation through the two filters.

This situation may be equivalent to saying that the magnetic flux is transferred (moved) from the dayside to the magnetotail, forming the "stretched" dipolar field lines in the anti-solar direction or in the tailward direction. When the IMF southward component is increased, the dynamo process, the resulting cross-tail current, and the tail field will increase. This is equivalent to an increase of the transfer of the field lines from the dayside to the tail; the increased tail current "erodes" the dayside of the magnetopause. When the IMF southward component of the IMF decreases, the dynamo power is reduced and thus the stretched field lines contract. 


\section{Magentopause and Tail}

Magnetic field line filter

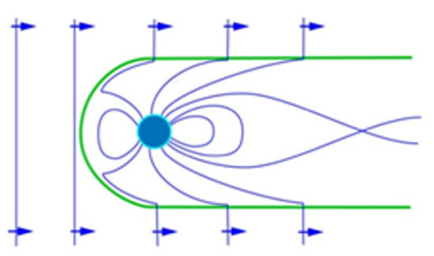

Electric current filter

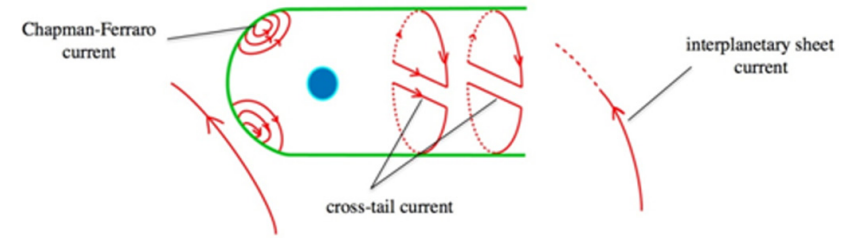

Fig. 8. The two views (magnetic field lines or electric currents) of the magnetopause and tail. The interplanetary current sheet will be discussed in Sect. 7.

This has an important consequence in initiating substorm onset (Sect. 4.2).

This consideration led us to an empirical determination of the power generated by the dynamo (cf. Akasofu, 1981), which was later confirmed theoretically by Pudovkin and Semenov (1986).

$\varepsilon=V B^{2} \sin ^{4}(\theta / 2) l^{2}$

The above empirical equation may be implicit in the moving field line concept, but the formulation of the dynamo power has so far not been explicitly demonstrated by the frozen-in field theory.

The solar wind-magnetosphere dynamo produces space charges, positive charges in the morning side, and negative charges in the evening side (see Fig. 9a). The electric field across the magneototail causes a large-scale convection of magnetospheric and ionospheric plasmas (Axford and Hines, 1961). It is this convection that produces the two eddy currents (called the DP2 current) in the ionosphere shown in the left side of Fig. 9b. The unloading component is associated with substorms and is discussed in the next section.

\section{Magnetospheric substorms}

Now, suppose that the dynamo power is increased to the level of $10^{18} \mathrm{erg} \mathrm{s}^{-1}$ ( $10^{11}$ watts) by an increase of $\theta$ and $B$, namely, the southward turning of the IMF. As mentioned above, the dynamo power $\varepsilon$ is increased as the Eq. (1) indicates. Subsequently, the two semi-circular currents and the magnetic flux in the tail will increase. This is equivalent to an increase of the transfer of magnetic flux from the dayside in the magnetotail.

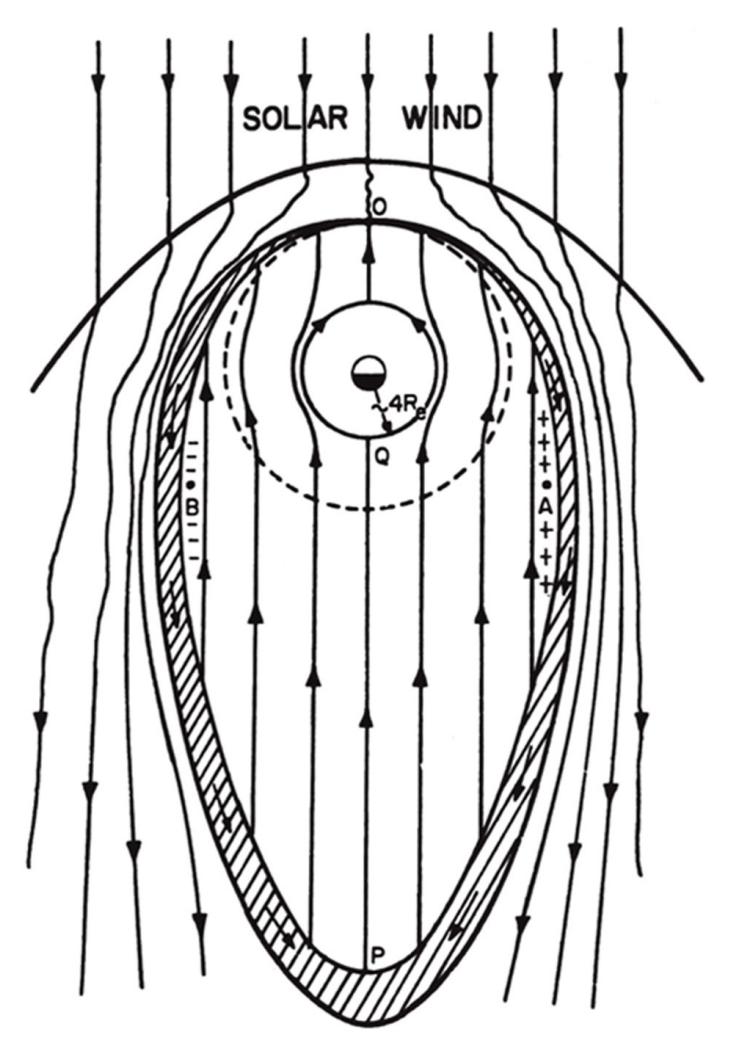

Fig. 9a. The magnetospheric dynamo produces a large convection of magnetospheric plasma and two eddy currents in the ionosphere, which are shown in Fig. 9b (Axford and Hines, 1961).

This increase of the power $\varepsilon$ increases first the directly driven current eddies in the ionosphere (see Fig. 9b, left, and Fig. 9c). This may be considered as a manifestation of the growth phase of substorms. The substorm current system is the unloading component, which tends to develop a little later (Fig. 9b, right). This will be discussed in Sect. 4.1.

\subsection{Bostrom's two substorm circuits}

The unloading component (or DP1 current) of the current system is actually a 3-D current system; Figs. 9c and $9 \mathrm{~d}$ indicate only the growth and decay of the ionospheric part of the currents. The unloading component model, namely, the substorm current system, was established by Bostrom (1964). It consists of two circuits, the meridional circuit and the azimuthal circuit; both are shown in Fig. 10. Both the directly driven and the unloading current systems grow after the dynamo power is increased (Figs. 9c and 9d). The directly driven current grows soon after the power $\varepsilon$ of the solar wind-magnetosphere dynamo is increased, while the unloading current grows impulsively after some time. In this paper, we are mainly concerned with the unloading component (substorm current system), namely, Bostrom's two currents.

We have made a long-time effort to confirm Bostrom's two currents by operating the Alaska meridian chain and the 
NORMALIZED PATTERNS 1000 -2000 UT ON MARCH 19, 1978

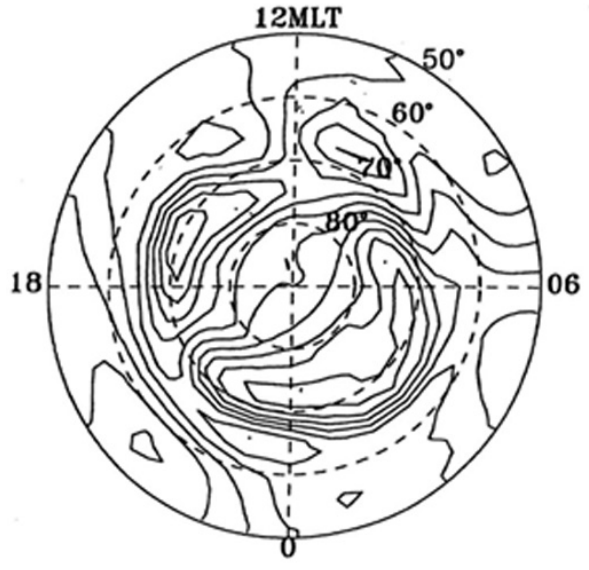

DIRECTLY DRIVEN COMPONENT

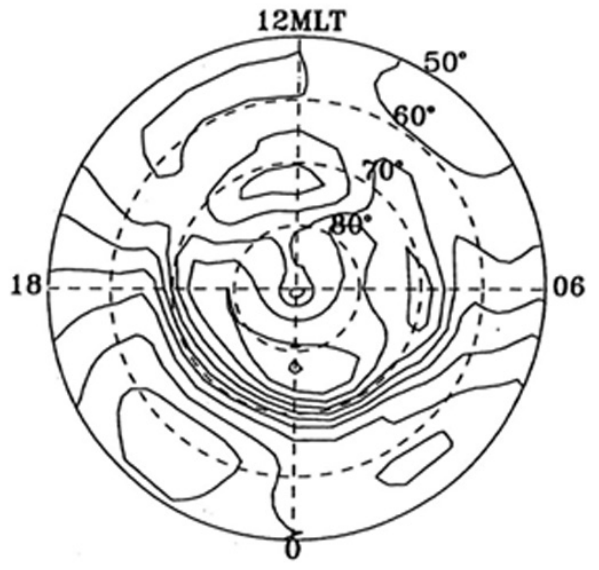

UNLOADING COMPONENT

Fig. 9b. The equipotential pattern of the directly driven current (left) and the unloading current (right); see Sun et al. (1998).

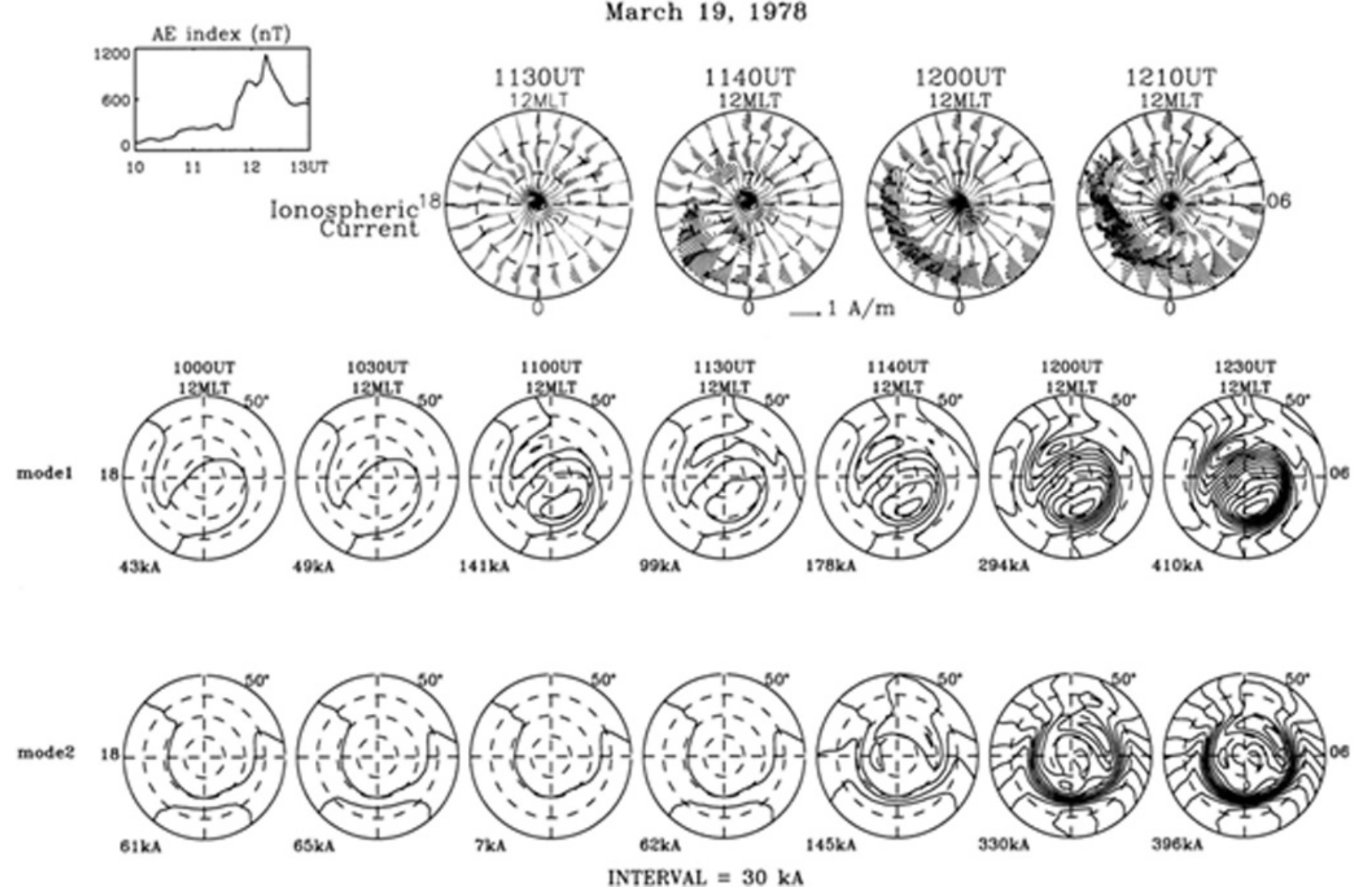

March 19, 1978

Fig. 9c. The growth of the ionospheric currents during a substorm. Top, the ionospheric current. Middle, the equipotential pattern of the directly driven current. Bottom, the equipotential pattern of the unloading current (Sun et al., 1998). 


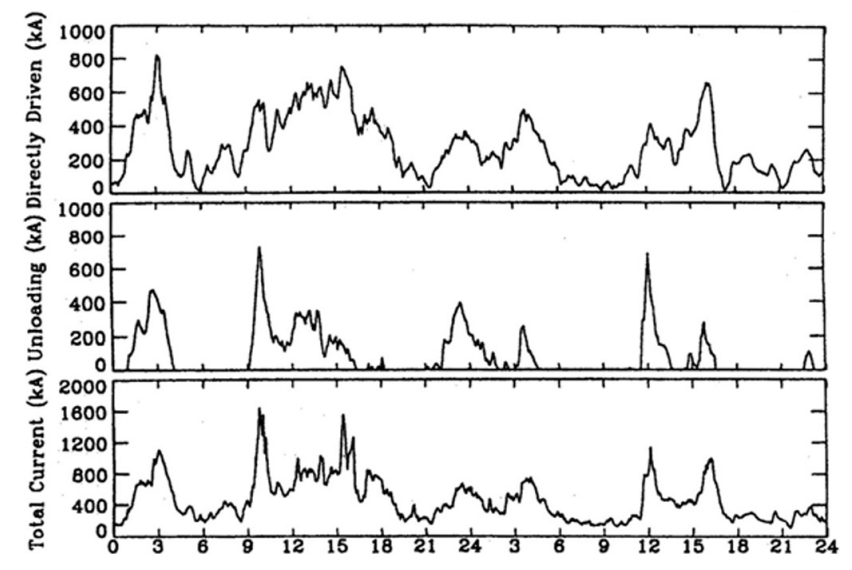

Fig. 9d. The growth and decay of the directly driven current (top), the unloading component (middle), and the total current (bottom) during a series of substorms (Sun et al., 1998).

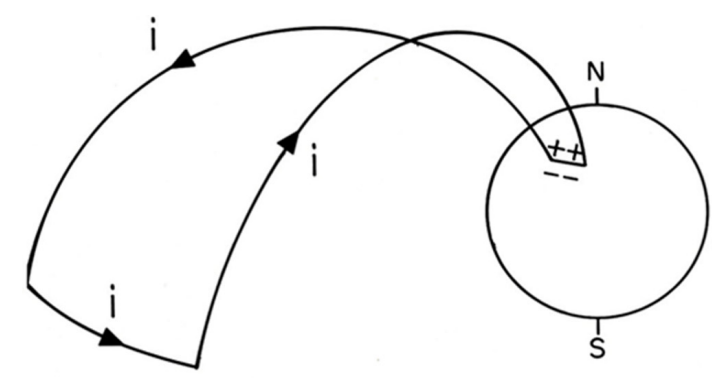

Azimuthal loop

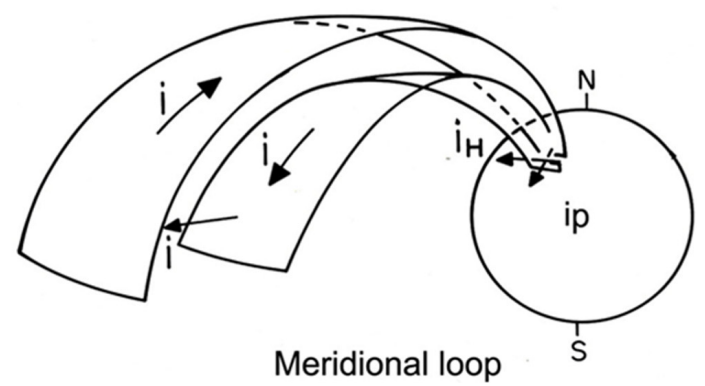

Fig. 10. Bostrom's azimuthal and meridional current loops (Bostrom, 1964).

International Six Meridian magnetometer chains and by analyzing the records on the basis of the KRM method. We show here an example of the method of proving the presence of the meridional circuit.

In proving the meridional circuit, we noted first of all that the Pedersen current in the ionosphere is connected to a pair of field-aligned current sheets (upward and downward) and the equatorial radial current, forming a loop. Kamide and Akasofu (1981) obtained the distribution of the Pedersen

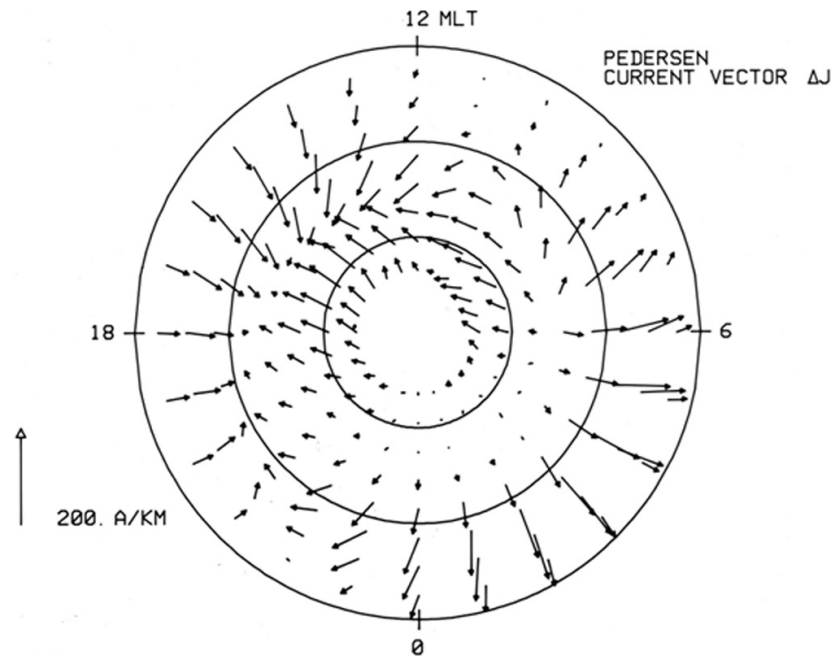

Fig. 11. The distribution of the Pedersen current (Kamide and Akasofu, 1981).

current vectors based on records from the Alaska meridian chain of magnetometers (Fig. 11) and "projected" them on the equatorial plane (Fig. 12, left; Akasofu et al., 1981). Fortunately, later Iijima et al. (1990) obtained the distribution of the radial current on the equatorial plane on the basis of the AMPTE satellite data (Fig. 12, right). Their data enabled Akasofu (1992) to compare the projected vector distribution with the satellite-based data. The comparison is shown in Fig. 12. Although both are long-time average data during different periods and are obtained by entirely different methods, the agreement is fairly good, proving the presence of Bostrom's meridional current loop current circuit, as well as the analysis method of the ground-based and satellite-based data.

The meridional component consists of two sheet currents, one away from the Earth and the other toward the Earth. The former, the upward current sheet carried by downwardmoving electrons, is responsible for forming auroral arcs (see Fig. 13a.).

Figure $13 \mathrm{~b}$ shows a segment of the meridional component.

There are two important implications in the above analysis. The first point is that along the meridional circuit the term $\boldsymbol{E} \cdot \boldsymbol{J}$ is negative only on the equatorial plane (except for the potential drop which accelerates auroral electrons), so that the meridional component must be driven by $\boldsymbol{E}$ on the equatorial region. Secondly, the location of the base of the observed radial vectors corresponds to the location where the upward part of the field-aligned current ends and the starting point of the electron beam to the auroral ionosphere. The significance of the distribution of the base of the radial current vectors in Fig. 12 is that the bases are distributed at distances as close as $L=4-5$. This suggests that the initially brightening auroral arc (IBA) at substorm onset can be connected to a distance as close as $L=4-5$ on the equatorial plane. 

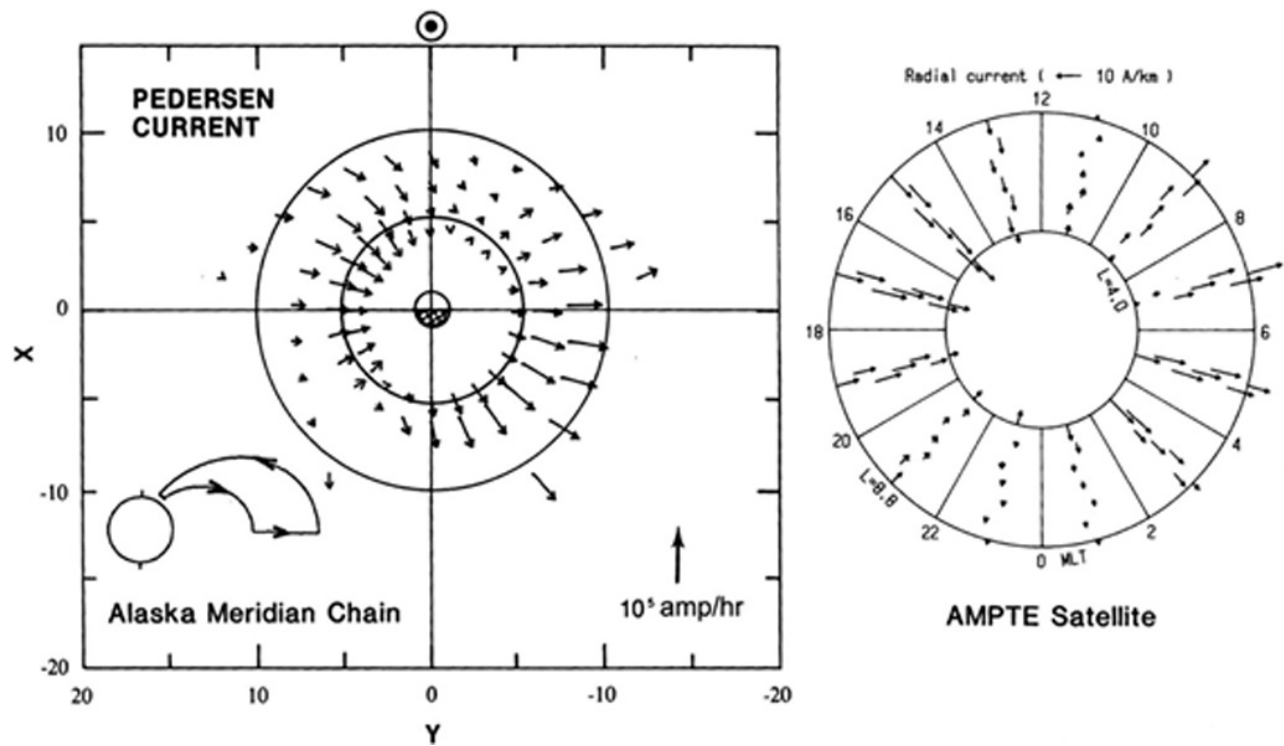

AMPTE Satellite

Fig. 12. The comparison of the Pedersen current projected on the equatorial plane and the observed radial current (Akasofu, 1992).

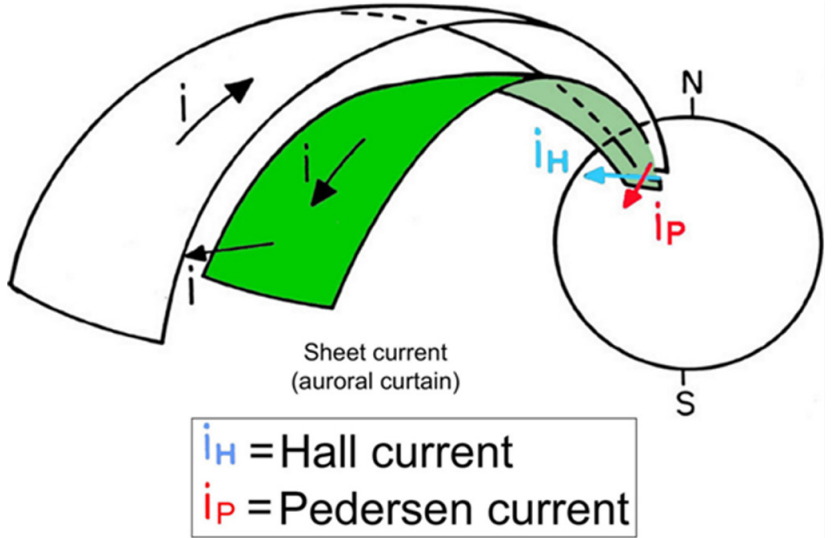

Boström's current (1964)

Fig. 13a. Bostrom's azimuthal current loop. It is a sheet form that produces the auroral curtain.

This is the first observational proof of the equatorial location connected to IBA. In fact, IBA is often observed at gm.lat. $60^{\circ}$. These facts are crucial in considering the process for substorm onset. Further, the Lorentz force $(\boldsymbol{J} \times \boldsymbol{B})$ may be responsible for the evening-morning asymmetry of auroral substorms, such as westward traveling surges in the evening sky and omega bands/torches in the morning sky.

Furthermore, the resulting electric field $\boldsymbol{E}$ drives not only the Pedersen current in the ionosphere, but also the westward electrojet, which is mainly the Hall current (Fig. 14). The electrons in the ionosphere flow along the potential contour lines of the unloading component (right side of Fig. 9). In fact, radar observations show that the westward electro-

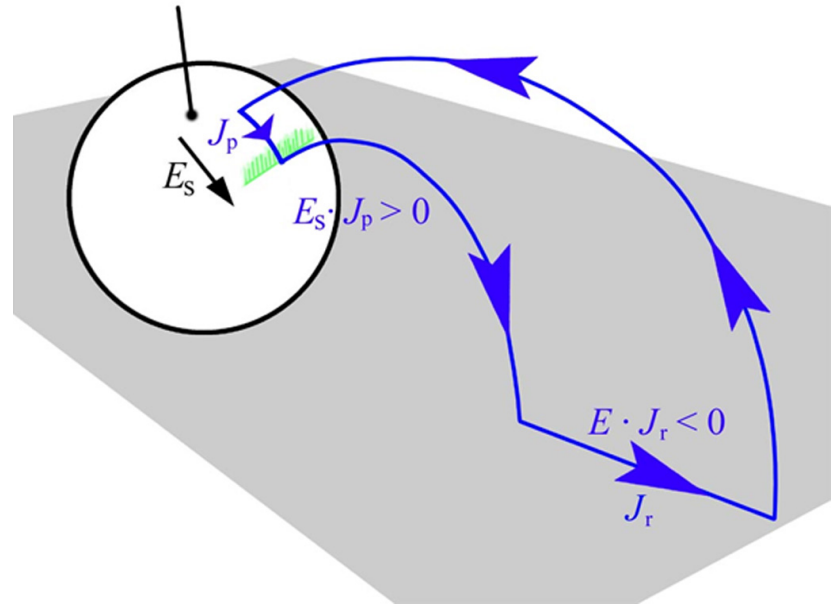

Fig. 13b. A segment of Bostrom's meridional circuit.

jet is mainly caused by the eastward flow of electrons. Further, radar observations do not show a westward electric field associated with the westward electrojet. Note that if the westward electrojet is caused by a diversion of the crosstail current, the cross-tail potential (or a westward electric field) should be continuously observed along the night side of the oval during substorms; however, this is not the case. Therefore, the westward electrojet is not caused by the socalled "current wedge", namely, the diversion of the crosstail current (Fig. 15a). The westward electrojet must be driven by the electric field $\boldsymbol{E}$. Thus the earthward electric field is shown to drive both Bostrom's meridional and azimuthal loops. 


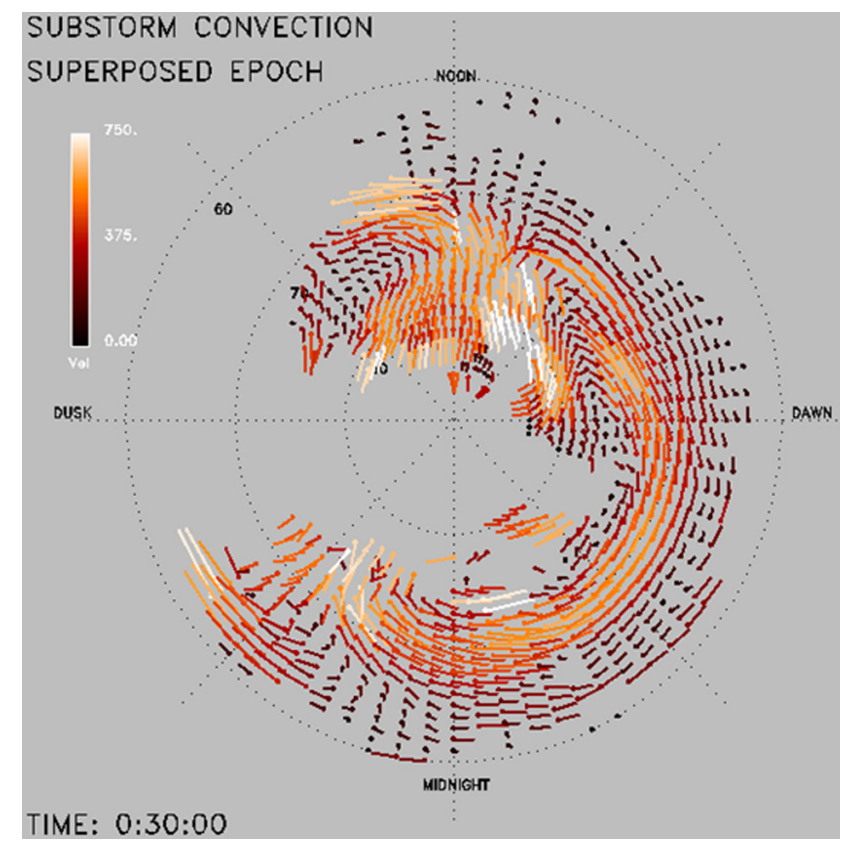

Fig. 14. The flow of electrons during a substorm. The eastward flow represents the westward electrojet (Bristow and Jensen, 2007).

It is expected that a positive feedback process occurs in order for a substorm to grow rapidly. Since the polar cap is not conductive enough, the westward electrojet cannot have its return current in the polar cap. Thus the zero divergence condition of current requires that the jet current closes in the magnetosphere. Figure 15 a schematically shows this situation, while Fig. 15b shows the projected vector of the ionospheric currents on the equatorial plane. The return current from the westward electrojet will reduce the cross-tail current, enhancing the separation of electrons and protons further in the plasma sheet and thus $\boldsymbol{E}$, which enhances further the westward electrojet as well (see Sect. 4.2 and Fig. 16).

The reason why the substorm current system is discussed in so much detail here is that Bostrom's current system can explain the two major features of substorms, the aurora (the meridional sheet current) and the westward auroral electrojet (azimuthal current) without invoking other processes.

Summarizing this subsection, it is shown that the study of the substorm current system here has led us to these conclusions:

1. The earthward electric field $\boldsymbol{E}$ at distances of $5-8 R_{\mathrm{E}}$ is the primary driving force of substorms. Thus a study of the substorm current system is essential in understanding substorm phenomena, rather than magnetic reconnection in the magnetotail.

2. The equatorial region which is connected to the initially brightening arc (IBA) can be located at distances as close as $4-5 R_{\mathrm{E}}$; see also Sect. 4.3.

\section{Growth Phase}

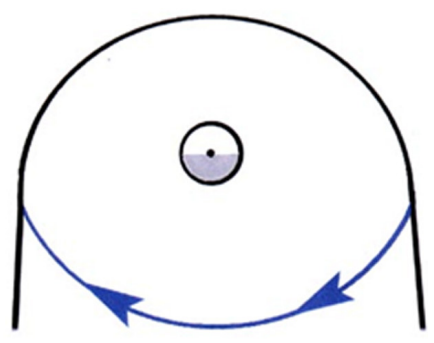

'Dipolarization'

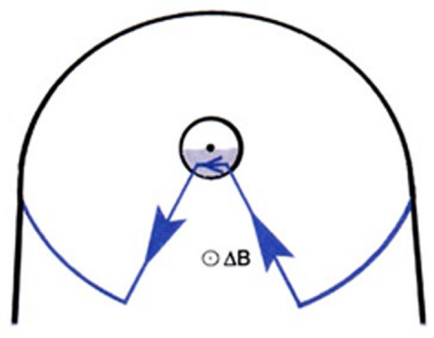

\section{Cross-tail current Disruption}

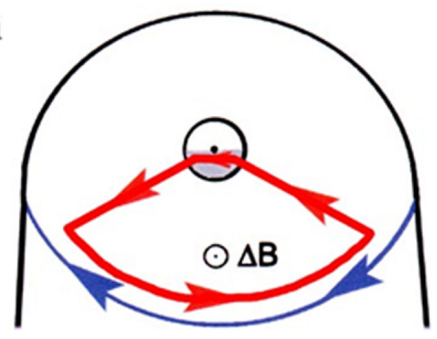

Fig. 15a. Schematic representation of changes of the cross-tail current. The top one shows the cross-tail current. The middle one suggests the diversion of the cross-tail current (so-called "current wedge"). The bottom one, same as the azimuthal current in Fig. 10, suggests the reduction of the cross-tail current caused by the return current of the westward electrojet.

3. The westward electrojet is not the diverted cross-tail current.

4. The return current from the westward electrojet reduces the cross-tail current, causing a positive feedback process for a rapid growth of substorms (see Sect. 4.2).

5. Although it is not shown here, the magnetic field produced by the azimuthal loop current is responsible for advancing the earthward end of the loop poleward, namely, poleward expansion of auroral substorms; magnetic reconnection and the dipolarization cannot provide enough magnetic flux for the poleward expansion of a few hundred kilometers. 
March 19, 1978
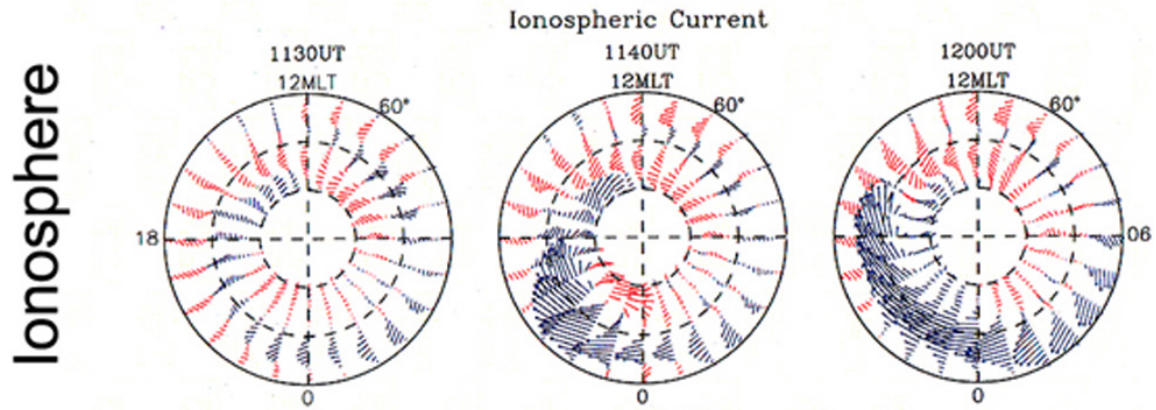

$\longrightarrow 1 \mathrm{~A} / \mathrm{m}$

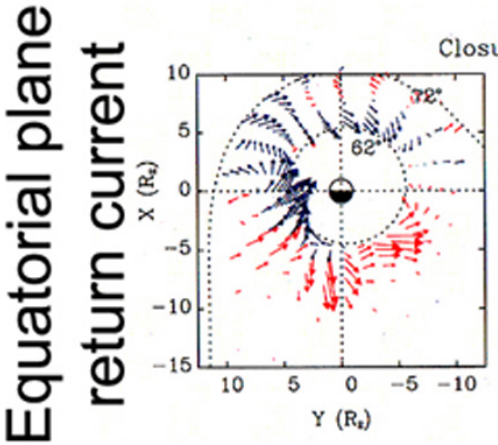

Closure Current the equatorial plane
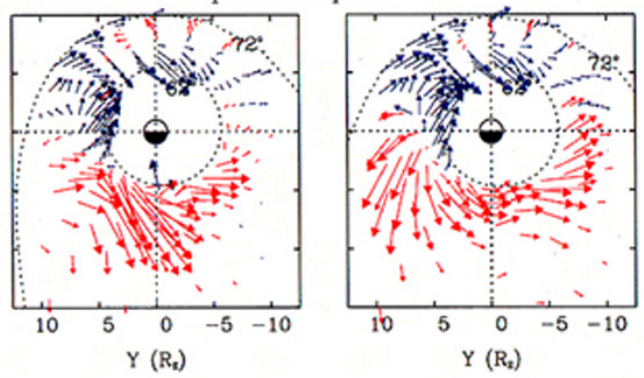

Fig. 15b. The possible return current distribution of the westward electrojet. The distribution of the ionospheric currents (upper) is projected on the equatorial plane (lower). In the lower part, the projected westward electrojet vectors are shown in red (cf. Akasofu, 2007, p. 106).

\subsection{A cause of substorms}

We learned in Sect. 4.1 that the growth of an earthward electric field $\boldsymbol{E}$ can explain some of the major features of auroral substorms and the substorm current system. One possible cause of substorms is as follows: If the southward IMF is reduced, the cross-tail current is reduced, and thus the "stretched" field lines in the magnetotail "contract", carrying electrons with them toward the Earth; however, protons, not gyrating around the magnetic field lines, will not participate in the earthward motion of the electrons. This process of separating electrons and protons is a dynamo process that produces the needed earthward $\boldsymbol{E} r$ that drives the Pedersen current in the ionosphere and powers the meridional circuit (Fig. 13b). In fact, a significant number of substorms are triggered by a northward turning of the IMF (Lyons et al., 2001). This process does not require magnetic reconnection and cannot be treated by the standard MHD theory, because protons in the plasma sheet are not frozen to the field lines. Further, there has so far been no explanation of substorms initiated by the northward turning IMF.

It can be seen that this way of consideration can provide a reasonable chain of processes leading to substorm onset without invoking magnetic reconnection in the magnetotail. Furthermore, the above consideration is based on a study of the substorm current system.

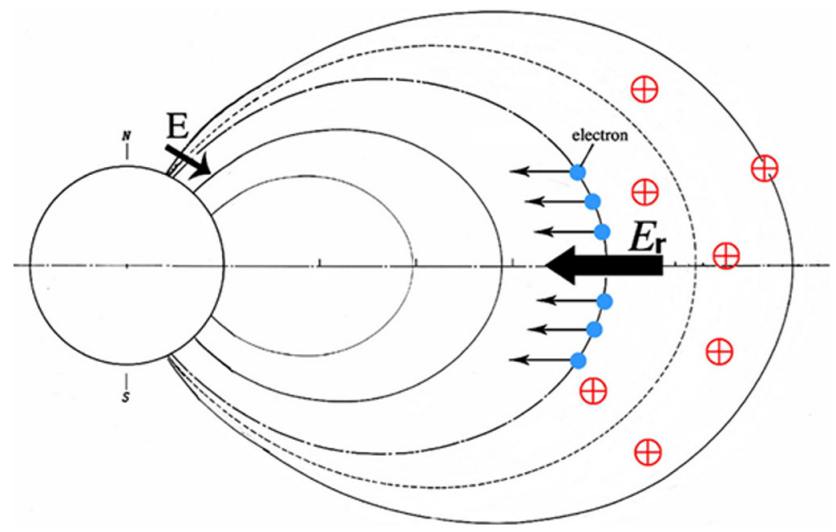

Fig. 16. Schematic illustration of how the earthward electric field $\boldsymbol{E} r$ can be produced when the cross-tail current is reduced (Akasofu, 2007, p. 117).

Substorms are a complex phenomenon. It is expected that there are other causes as well. When the southward component of the IMF is large and steady for more than $10 \mathrm{~h}$, there occurs a series of substorms; if some plasma instability occurs in the cross-tail current and reduces its intensity (cf. Lui, 2004), the same process mentioned above could occur. In any case, it is crucial to search for the cause of the earthward electric field in understanding substorm phenomena. 


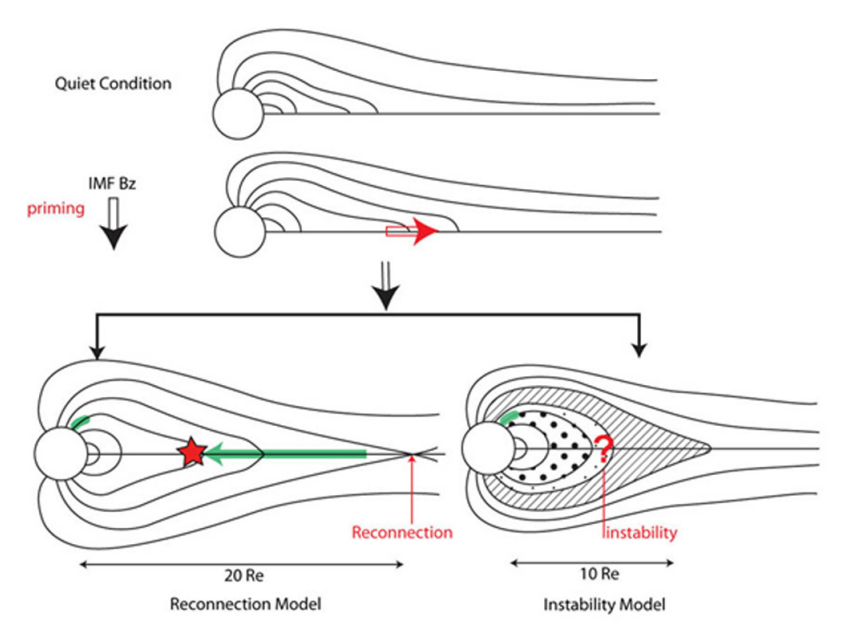

Flow Energy Magnetic Energy Dynamo Process

Fig. 17a. Schematic illustration of two models which cause substorms.

\subsection{Examination of the magnetic reconnection theory by an auroral observation}

It is almost universally believed that magnetospheric substorms are caused by magnetic reconnection at a distance of about $20 R_{\mathrm{E}}$ in the magnetotail. It appears that many researchers, theorists and observers alike, are bound to prove solely this premise. Alfvén was seriously concerned about this one-sided trend (Alfvén, 1986, p. 786). Because of this theoretical premise, an earthward plasma flow from $20 R_{\mathrm{E}}$ is needed to trigger substorm onset deep in the magnetosphere (Fig. 17a); many simulation and observational studies are focused only on this process. However, although some fast flows are observed in the magnetotail, their relation to substorm onset has not definitely been confirmed.

On the other hand, it is shown that a reduction of the crosstail current can produce the electric field that can trigger substorm onset. In fact, the northward turning or a reduction of the southward component of the IMF is the only definitely known external signal to be related to substorm onset (Lyons et al., 2001).

There is a small minority group that believes that substorms are initiated by a process within a distance of about $10 R_{\mathrm{E}}$ (Fig. 17a); see Henderson (2009). Thus a test was conducted on the basis of auroral morphology. If the earthward flow of plasma is responsible for IBA, it should activate auroral arcs located poleward of the IBA before substorm onset. However, it was found that the poleward arcs may be activated only after, not before, substorm onset (Fig. 17b) (see Akasofu et al., 2010). Therefore, although this test is not the ultimate one, it suggests that substorms are initiated internally, not externally as the reconnection theory suggests. It may be added that the above discussion does not exclude magnetic reconnection as a secondary process.
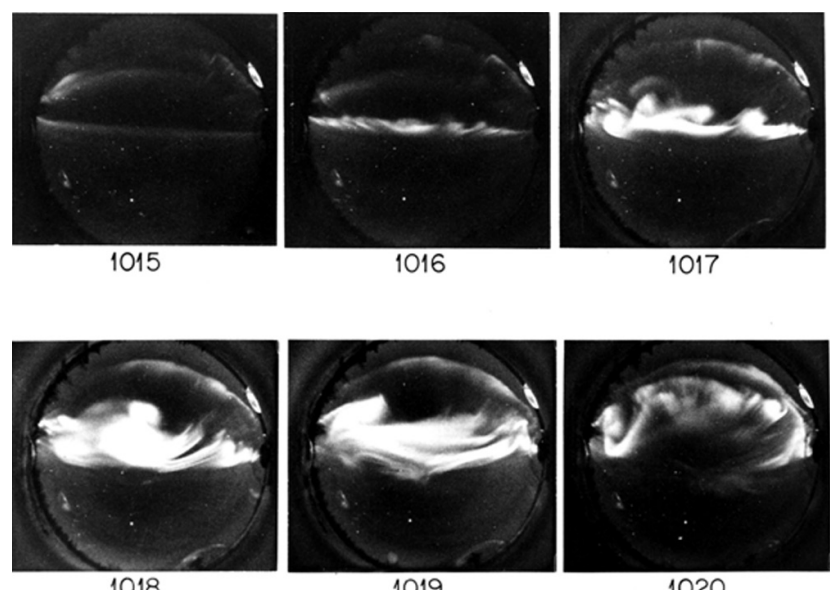

1019

NOVEMBER 20, 1976

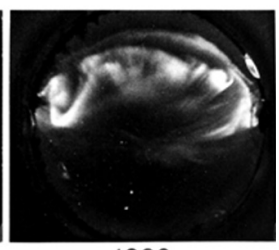

1020

Fig. 17b. An example of substorm onset as shown by a sudden brightening of an arc. An arc located poleward of the initially brightening arc shows some activity after substorm onset (Akasofu et al., 2010).

\section{SOLAR FLARES}
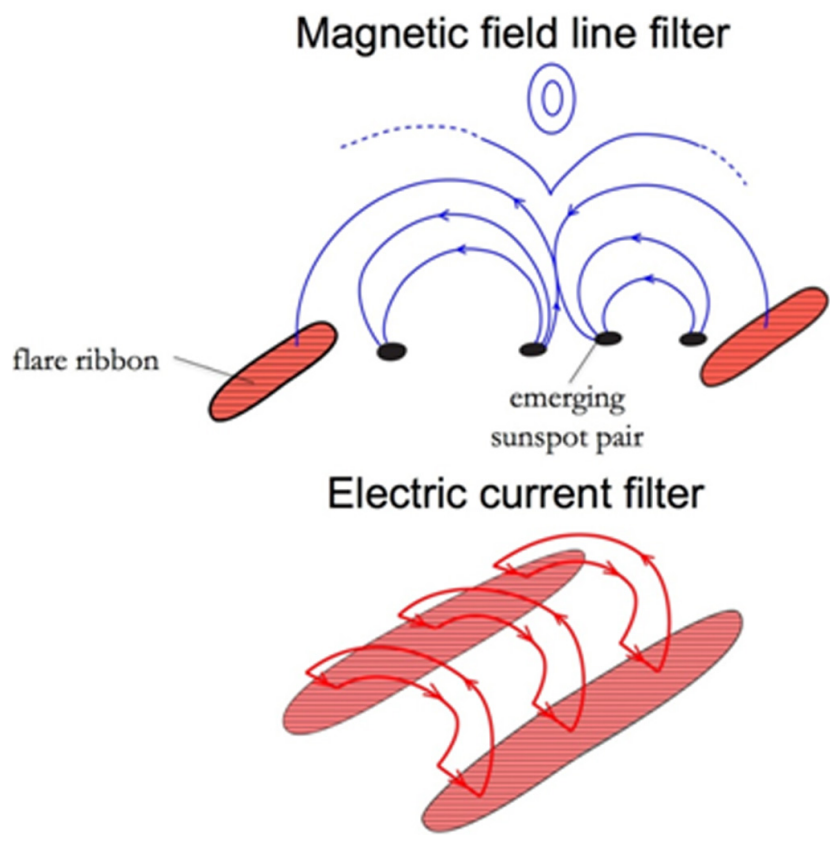

Fig. 18. The two views (magnetic field lines or electric currents) of a solar flare.

\section{Solar flares}

It has been almost universally believed that magnetic reconnection causes solar flares when two sunspot pairs come into contact (Fig. 18), although a few exceptions deal with an instability of magnetic flux ropes (cf. Titov and Demoulin, 1999; Chen and Krall, 2003). 


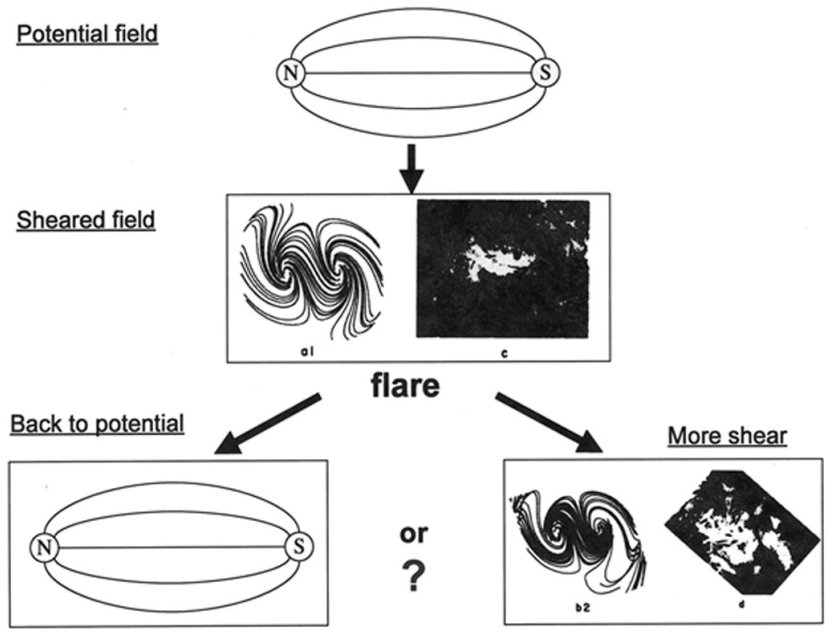

Fig. 19. Schematic illustration of two possibilities, depending on how magnetic energy changes during a flare.

In this paper, we examine solar flares from an observational point of view. First of all, it is important to note that with respect to Fig. 6a, Sheeley et al. (1975) mentioned that “ - reconnections occur much more often than flares, and thus usually occur without them."

In the solar corona, magnetic energy of a force-free field, namely, the field of $\boldsymbol{i} \times \boldsymbol{B}=0$, is available. In solar physics, this type of field is expressed in terms of the "sheared" field; the shear signifies deviation from a potential field (or distortion of the field lines from the potential field lines by fieldaligned currents). The sheared field contains expendable energy for solar flares.

Thus it is interesting to test how the degree of shear will decrease during solar flares, indicating that the magnetic energy in the force-free field is expended (Fig. 19). This test showed that, without exception, the shear increases at flare onset time (Fig. 20) (Wang et al., 1994). This fact indicates that magnetic shear must be increased above some critical level before and during flare onset. A dynamo process associated with motions of photospheric gas may be needed (Kan et al., 1983; Akasofu, 1984). In fact, a rapid rotation of one of the sunspots in the pair was observed during a recent typical flare (Fig. 21). Title (2007) described this phenomenon as "a kind of magnetic hurricane."

The result shown in Fig. 20 is contrary to what is expected from the magnetic reconnection (annihilation) hypothesis. The magnetic energy unexpectedly increased rather than decreased. This condition is similar to that of magnetospheric substorms. In the early days, when the magnetotail was discovered, it was thought that the magnetotail had enough magnetic energy for many substorms, but it soon became apparent that the magnetosphere has to be "primed" by the southward IMF prior to substorms. Increased power $\varepsilon$ of the solar wind-magnetosphere dynamo is needed by an increase of the southward component of the IMF.

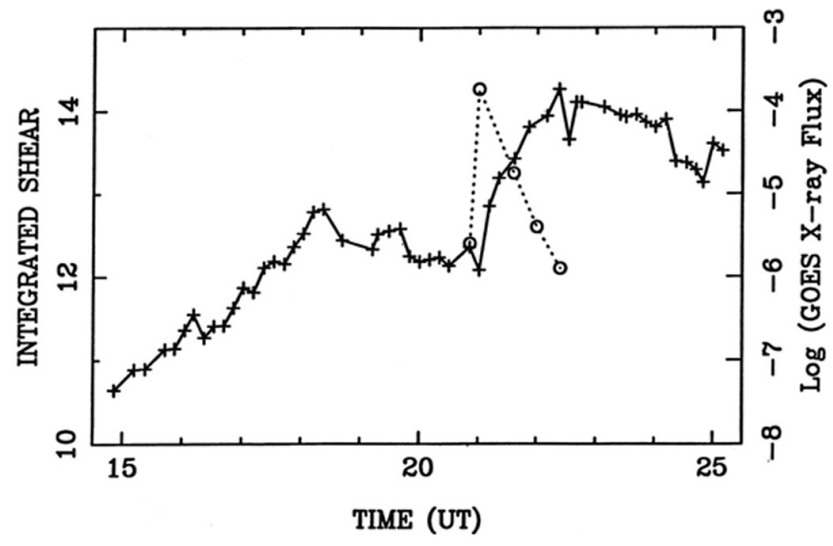

Fig. 20. A typical example of an increase of magnetic shear during a flare (Wang et al., 1994).

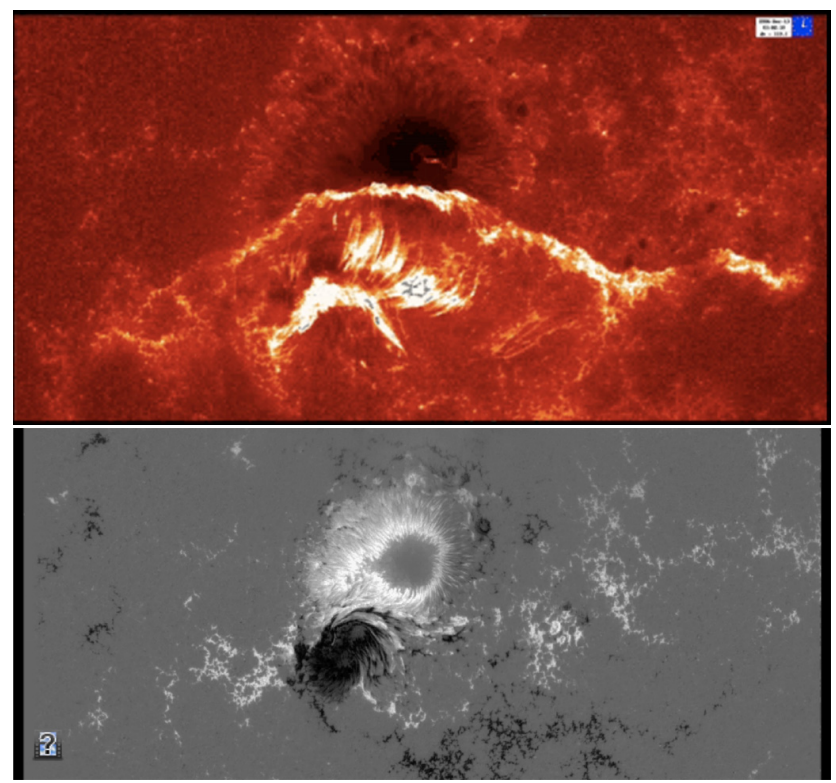

Fig. 21. In this sunspot pair, the negative spot rotated during a flare on 13 December 2006 (courtesy of K. Shibata).

This example indicates that one should consider the whole system, including a dynamo which increases the resultant currents along magnetic field lines.

It is well known that a typical flare can occur even without sunspots or a colliding sunspot pair (Fig. 22a). Indeed, in an arch-like magnetic configuration, a typical two-ribbon flare can occur. In such a case, it is expected that there occurs a shear flow along the central line of the arch-like structure, which acts as a dynamo. The resulting currents along the arch-like structure can cause a two-ribbon flare (Choe and Lee, 1995). This simplest flare demonstrates that magnetic reconnection caused by an emerging sunspot pair is not needed in causing solar flares and also that the dynamo process is needed. At the top of the magnetic arch, magnetic 


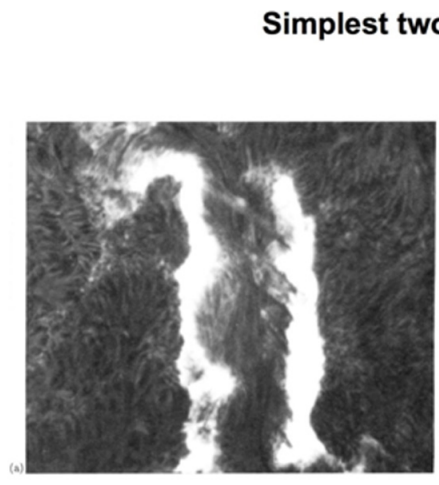

Zirin, 1988

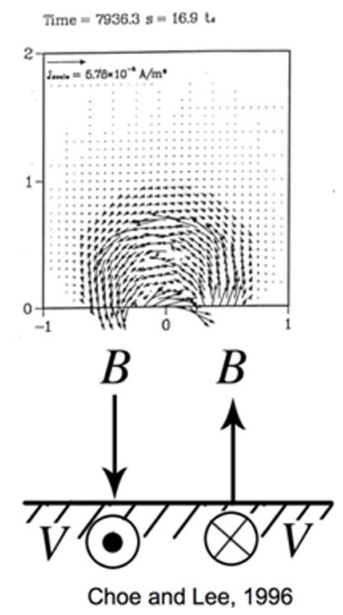

Fig. 22a. A typical two-ribbon flare without colliding sunspot pair (courtesy of H. Zirin) and the current system around the magnetic arch (Choe and Lee, 1995).

reconnection and various dynamical processes can occur, but their contributions to flare processes are not clear.

To understand a very complex flare (cf. Fig. 22b), it may be worthwhile to divide such a flare region into several simple magnetic arch (two-ribbon flare) regions, instead of paying too much attention to sunspots. In any case, it may well be that the two-ribbon flare (Fig. 22a) is the simplest case, the fundamental element of a solar flare. It is for this reason that the current filter can see only the currents along the magnetic arch (Fig. 18). Indeed, an arch-like structure is quite common in complex flares (cf. Fig. 21).

\section{Coronal mass ejections}

It has been shown by Burlaga et al. (1981) that magnetic clouds in coronal mass elections (CMEs) contain a helical magnetic structure, indicating the presence of electric currents along the loop. The purpose of this paper is not to discuss in detail the magnetic structure of CMEs. It is to point out how much electric currents are contained in magnetic clouds. Figure 23a simulates such a magnetic configuration, and Fig. 23b shows the observed and simulated changes of the solar wind, indicating that the simulation model is a reasonable one. Gosling et al. (1986) also found bi-directional flows of energetic electrons in some loop-like configurations, suggesting that both feet of the loop are embedded in the photosphere. The current in the loop is estimated to be $10^{9}$ amperes (Fig. 24), assuming that a hypothetical cylinder of magnetic cloud of radius of 0.2 au passes by the Earth in $12 \mathrm{~h}$ with a speed of $700 \mathrm{~km}$; the observed IMF is about $10 \mathrm{nT}$. Again, it is important to note that flare processes must generate such electric currents; the total current involved may be substantially greater and thus the question is how it is gen-
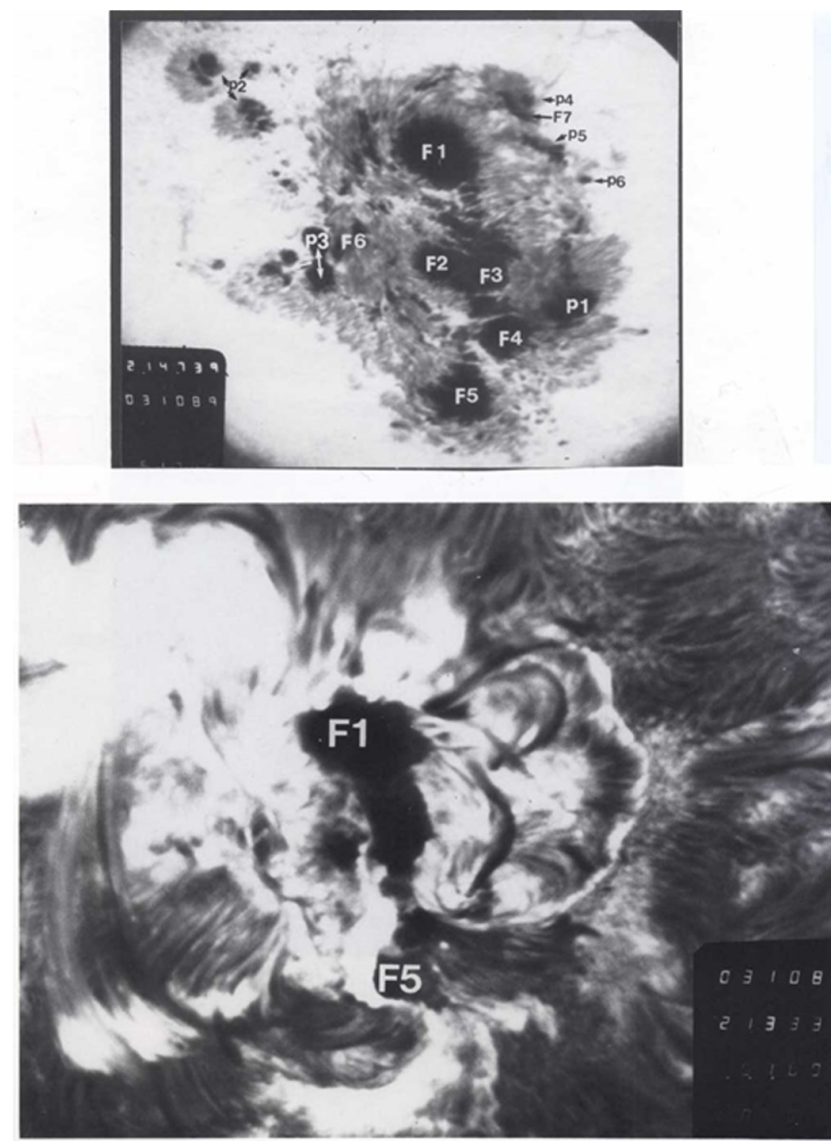

Fig. 22b. A typical complex flare (courtesy of the Big Bear Solar Observatory).

erated. As examples, Titov and Demoulin (1999) estimated it to be $7 \times 10^{12}$ amperes and Chen and Krall (2003) considered it to be $10^{11}$ amperes.

\section{The interplanetary magnetic field}

It is known that the "warped" equatorial current sheet extends from the Sun to interplanetary space (Fig. 25); the IMF field lines have a spiral structure on it. As the Earth crosses this current sheet, the polarity of the IMF changes from "away" to "toward" or vice versa. This is because the current sheet separates the northern and southern IMF from the Sun. Since the divergence of $\boldsymbol{B}$ must be zero, it is important to find the entire configuration of the IMF in the heliosphere. The spiral IMF lines must be produced by currents that are perpendicular to the IMF lines (Fig. 26).

It is possible to infer the 3-D configuration of the electric current system in the heliosphere, assuming it to be a spherical, on the basis of unipolar induction associated with the rotating Sun (Alfvén, 1950, 1977, p. 279). When the solar dipole moment is oriented southward, the radial currents from both poles flow to the northern and southern polar 


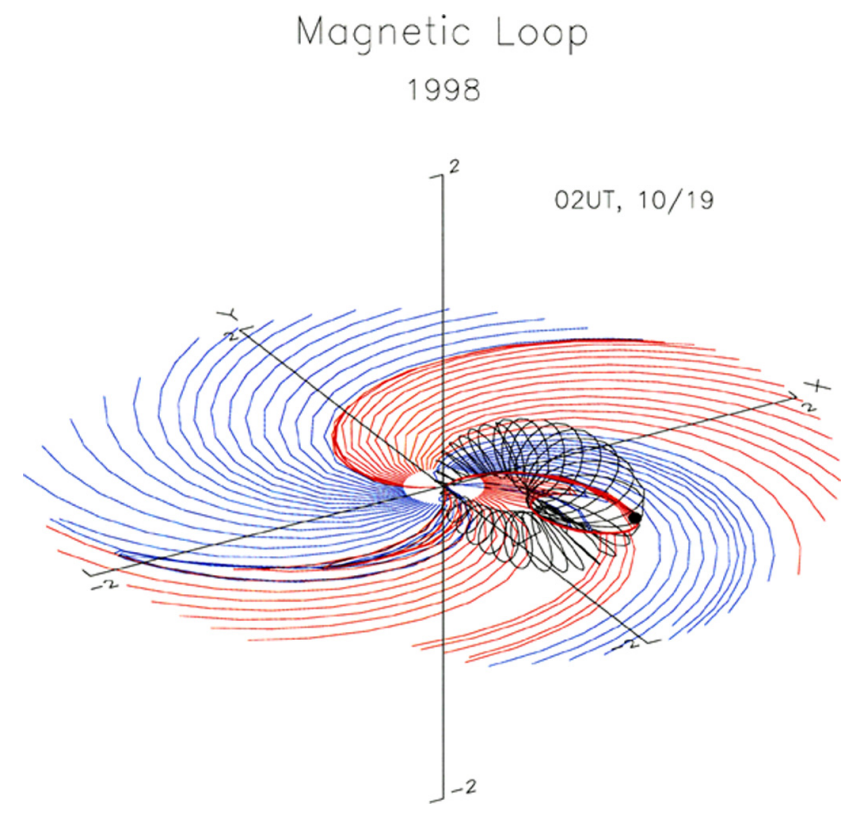

Fig. 23a. Some CMEs contain a helical magnetic structure (Saito et al., 2007).

boundaries of the heliosphere, respectively, and flow toward the equatorial plane along the boundary of the heliosphere; subsequently, the currents flow radially back toward the Sun (Fig. 27a). At the same time, a westward azimuthal current is generated on the equatorial plane. Both the radial current and azimuthal current on the equatorial plane can produce the spiral IMF field; the combined current is perpendicular to the spiral field lines. Note that the non-divergence of both $\boldsymbol{B}$ and $\boldsymbol{i}$ are maintained in this model. It is possible to compute the IMF in the heliosphere on the basis of the currents shown in Fig. 27a. Figure 27b shows field lines in the heliosphere from solar latitudes $70^{\circ}$ and $80^{\circ}$. Ulysses' observations seem to confirm such a spiral feature.

\section{Summary}

1. Both the magnetic field line filter and the electric current filter are needed in advancing our field beyond Dungey's magnetic reconnection theory.

2. The current line filter leads us to the need for a study of dynamo processes which can supply the power for solar and magnetospheric processes.

3. The present study of the substorm current system leads us to find that an earthward electric field at distances 5$8 R_{\mathrm{E}}$ is the primary driving force of substorms, rather than magnetic reconnection at a distant magnetotail.

4. Solar flares associated with a magnetic arch structure without sunspots may be the most elementary flares.

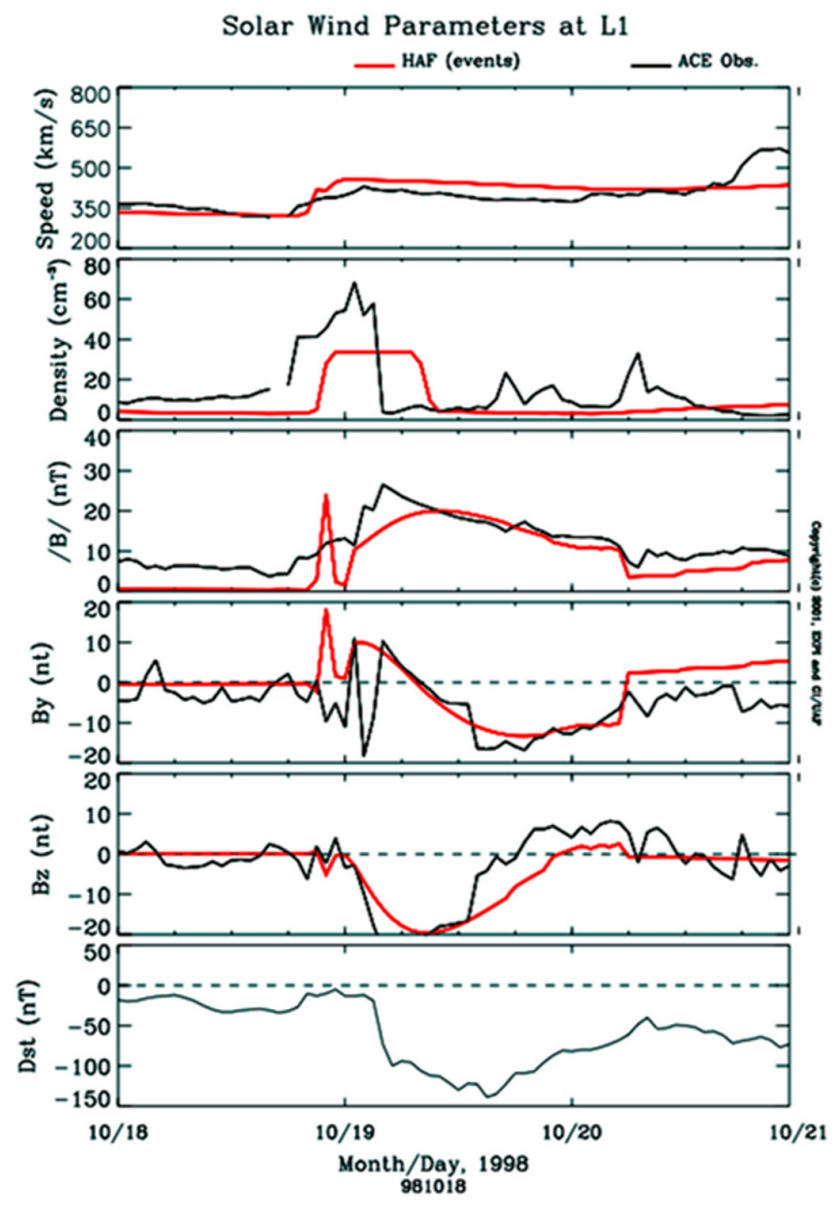

Fig. 23b. The observed magnetic field changes and the simulated changes based on the model shown in Fig. 23a (Saito et al., 2007).

5. Some magnetic clouds contain $10^{9}$ amperes of currents.

6. The unipolar induction theory enables us to compute the magnetic field line structure in the heliosphere.

\section{Concluding remarks}

In the history of science, there are periods when there is a high degree of agreement, both on theory and on problems to be solved within the framework provided by the theory. In such a situation, the theory tends to become the only one to be pursued, and other ideas tend to be excluded, or ignored. Observations that do not fit the theory are overlooked, ignored, or discredited. This is like a case in which most researchers believe that a particular jigsaw puzzle they are working is only one (say, a cat puzzle) and do not pay any attention to other possible puzzles.

Such a situation could actually delay progress in a field, although the particular theory can be polished endlessly. Parker's theory of the solar wind (Parker, 1958) was a great first step. However, his nozzle theory became so popular that 


\section{CME}

\section{Magnetic field line filter}

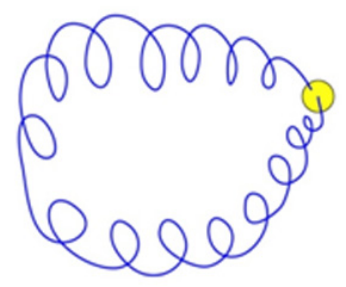

Electric current filter

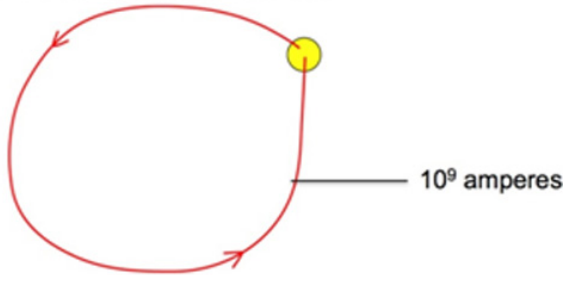

Fig. 24. The two views (magnetic field lines or electric currents) of a CME.

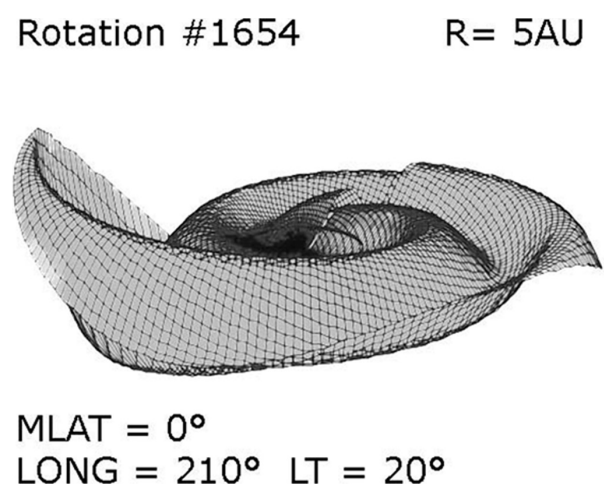

Fig. 25. The warped current sheet to a distance of 5 au (Akasofu and Fry, 1986).

early observations of the temperature profile in the corona, which did not support the theory, were ignored for many years. We are still at a loss as to the cause of the solar wind (cf. Tu et al., 2005), which is one of the most important phenomena in solar-terrestrial physics.

In order to achieve healthy advancement, we need to accommodate other ideas and to have debates, particularly when there is at least one piece (an "odd" piece) that does not fit in the presently working puzzle. If the "odd" piece is found to be genuine and similar pieces can be discovered, there is a good possibility that the subject of the presently working puzzle may have been misidentified (say, actually a dog puzzle).

This is one way by which our natural sciences can make a distinct advance. Young researchers are encouraged to find

\section{IMF and Current Sheet}

Magnetic field line filter
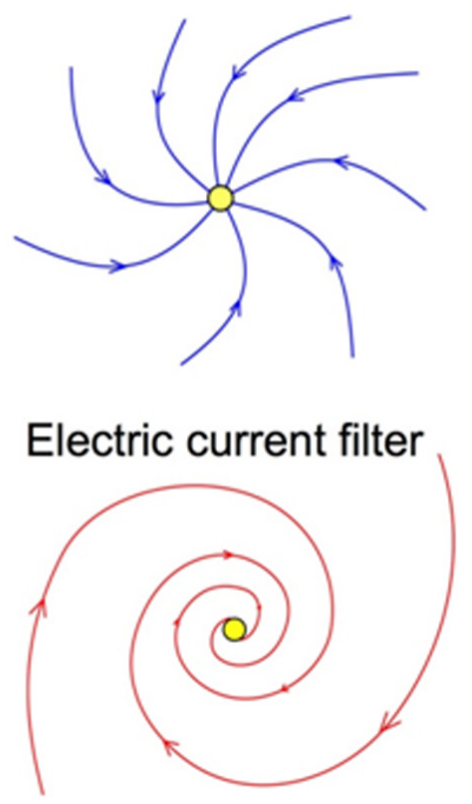

Fig. 26. The two views (magnetic field lines or electric currents) of the interplanetary system.

(a)

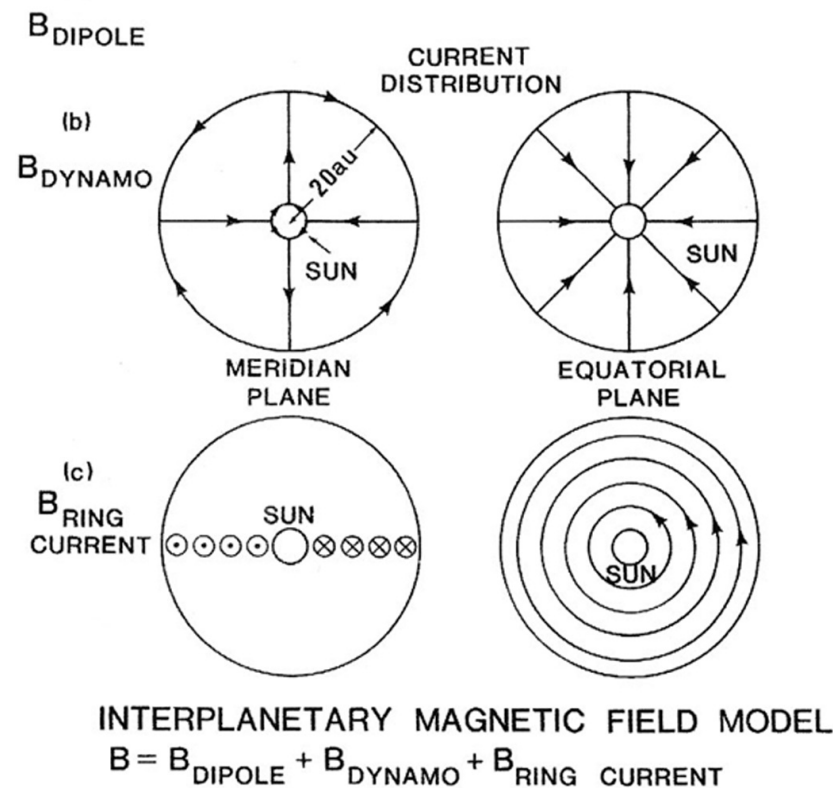

Fig. 27a. Schematic illustration of the interplanetary current system.

such "odd" pieces, although it may be hard to convince skeptical (naturally) colleagues at first. In this way, they can make a great contribution to their field. In this paper, the subject 

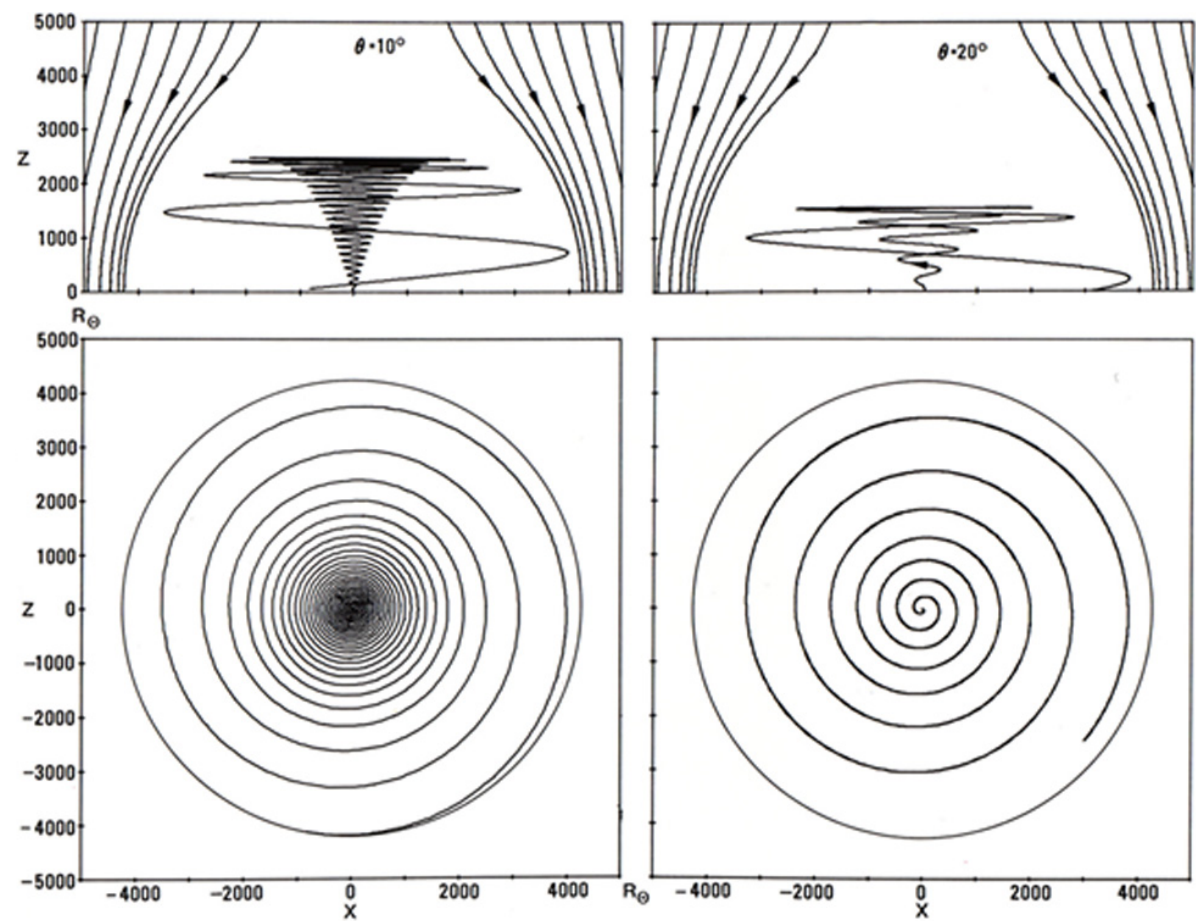

Fig. 27b. The interplanetary magnetic field lines, originating at polar angles of $10^{\circ}$ and $20^{\circ}$, in the heliosphere, computed on the basis of Fig. 24a (Akasofu and Covey, 1981).

of sunspots is intentionally used to illustrate this process, although it is not the ultimate solution. The other observational examples are also considered to provoke new ways of thinking. It is up to young researchers to find out if they are useful in advancing new ideas. In any case, through my research life, this is one important lesson I learned.

Acknowledgements. Looking back on my research life of about 50 years, I am very fortunate to have had crucial guidance by both Sydney Chapman and Hannes Alfvén. Alfvén gave me a way to study natural phenomena as a current system. I would like to thank all the researchers, young and old, who have advanced so many substorm studies. I would like to thank also my colleagues for participating in the six International Meridian Chains of Magnetometers. The National Science Foundation and NASA provided me with research funds for a long time.

Topical Editor I. A. Daglis thanks J. Burch for his help in evaluating this paper.

\section{References}

Akasofu, S.-I.: Energy coupling between the solar wind and the magnetosphere, Space Sci. Rev., 28, 121-190, 1981.

Akasofu, S.-I.: An essay on sunspots and solar flares, Planet. Space Sci., 32(11), 1469-1496, 1984.

Akasofu, S.-I.: Confirmation of the validity of the electric current distribution determined by a ground-based magnetometer network, Geophys. J. Inst., 109, 191-196, 1992.
Akasofu, S.-I.: Exploring the Secrets of the Aurora, Springer, 288 pp., 2007.

Akasofu, S.-I. and Chapman, S.: Solar-Terrestrial Phys., Oxford University Press, 901 pp., 1972.

Akasofu, S. I. and Covey, D. N.: Magnetic field configuration of the heliosphere in interstellar space, Planet. Space Sci., 29, 313-316, 1981.

Akasofu, S.-I. and Fry, C. D.: Heliospheric current sheet and its solar cycle variations, J. Geophys. Res., 91, 13679-13688, 1986.

Akasofu, S.-I., Kamide, Y., Kan, J. R., Lee, L. C., and Ahn, B.H.: Power transmission from the solar wind-magnetosphere dynamo to the magnetosphere and to the ionosphere: Analysis of the IMS Alaska meridian chain data, Planet. Space Sci., 29, 721 730, 1981.

Akasofu, S.-I., Lui, A. T. Y., and Meng, C.-I.: Importance of auroral features in the search for substorm onset processes, J. Geophys. Res., 115, A08218, doi:10.1029/2009JA014960, 2010.

Alfvén, H.: Cosmical Electrodynamics, Oxford, 237 pp., 1950.

Alfvén, H.: The theory of magnetic storms and aurorae, Nature, 167, 984-985, 1951.

Alfvén, H.: The second approach to cosmical electrodyamics, The Birkeland Symposium on Aurora and Magnetic storms, Sandefjord, Norway, 18-22 September 1967, edited by: Egeland, A and Holled, H., Centre National de la Recherche Scientifique, pp. 439-444, 1967.

Alfvén, H.: Electric currents in cosmic plasma, Rev. Geophys. Space Phys., 15(3), 271-284, 1977.

Alfvén, H.: Cosmic Plasma, D. Reidel Pub. Co., Dordrecht, Holland, 164 pp., 1981.

Alfvén, H.: Double layers and circuits in astrophysics, IEEE Trans, 
Plasma Sci., PS-14(6), 271-284, 1986.

Axford, W. I. and Hines, C. O.: A unifying theory of high-latitude geophysical phenomena and geomagnetic storms, Can. J. Phys., 39, 1433-1464, 1961.

Babcock, H. W.: The topology of the sun's magnetic field and the 22-year cycle, Astrophys. J., 133, 572-587, 1961.

Babcock, H. W. and Babcock, H. D.: The sun's magnetic field, 1952-1954, Astrophys. J., 121, 349-366, 1955.

Bostrom, R.: A model of the auroral electrojets, J. Geophys., 69, 4983-4999, 1964.

Bristow, W. A. and Jensen, P.: A superposed epoch study of SuperDARN convection observations during substorms. J. Geophys. Res., 112, A06232, doi:10.1029/2006JA012049, 2007.

Burlaga, L., Sittler, E., Mariani, F., and Schwenn, R.: Magnetic loop behind an interplanetary shock: Voyager, Helios, and IMP 8 Observations, J. Geophys. Res., 86, 6673-6684, 1981.

Chapman, S.: The theory of magnetic storms and aurorae, Nature, 186, 86, 1951.

Chen, J. and Krall, J.: Acceleration of coronal mass ejections, J. Geophys. Res., 108, 1410, doi:10.1029/2003JA009849, 2003.

Choe, G. S. and Lee, L. C.: Evolution of solarmagnetic arcades. II. Effect of resistivity and solar eruptive processes, Astrophs. J., 472, 372-388, 1995.

Cowling, T. G.: Solar electrodynamics, The Sun, edited by: Kuiper, G. P., pp. 532-591, 1953.

Deng, N., Liu, C., Yang, G., Wangand, H., and Dunker, C.: Rapid penumbral decay in active region NOAA 9026, associated with an X2.3 flare, Astrophys. J., 623, 1195-1201, 2005.

Dungey, J. W.: Interplanetary magnetic field and the auroral zones, Phys. Rev. Lett., 6, 47-48, 1961.

Gosling, J. T., Baker, D. N., Bame, S. J., and Zwickl, D. Z.: Bidirectional solar wind electron heat flux and hemispherically symmetric polar rain, J. Geophys. Res., 91, 11352-11358, 1986.

Henderson, M. G.: Observational evidence for an inside-out substorm onset scenario, Ann. Geophys., 27, 2129-2140, doi:10.5194/angeo-27-2129-2009, 2009.

Iijima, T., Potemra, T. A., and Zanetti, L. J.: Large-scale characteristics of magnetospheric equatorial currents, J. Geophys. Res., 95, 991-999, 1990.
Kamide, Y. and Akasofu, S.-I.: Global distribution of the Pedersen and Hall Currents and the equipotential pattern, during a moderately disturbed period, J. Geophys. Res., 86, 3665-3668, 1981.

Kan, J. R., Akasofu, S.-I., and Lee, L. C.: A dynamo theory of solar flares, Solar Phys., 84, 153-167, 1983.

Lui, A. T. Y.: Potential plasma instabilities for substorm expansion onset, Space Sci. Rev., 113, 127-206, 2004.

Lyons, L. R., McPherron, R. I., Zesta, E., Reeves, G. D. Sigwarth, J. B., and Frank, L. A.: Timing of substorm signatures during the November 24, 1996, Geospace environment modeling event, J. Geophys. Res., 106, 349-359, 2001.

Parker, E. N.: Dynamics of the interplanetary gas and magnetic fields, Astrophs. J., 128, 664-676, 1958.

Pudovkin, M. I. and Semenov, V. S.: Implication of the stagnation line model for energy input through the dayside magnetosphere, Geophys. Res. Lett., 13, 213-216, 1986.

Saito, T., Sun, W., Deehr, C. S., and Akasofu, S.-I.: Transequatorial magnetic flux loops on the sun as a possible new source of geomagnetic storms, J. Geophys. Res., 1112, AO5102, doi:10.1029/2006JA011941, 2007.

Sheeley, N. R., Bohlin, J. D., Bruecker, G. E., Purcell, G. E., Scherrer, J. D., and Tousey, V. E.: The reconstruction of magnetic field lines in the solar corona, Astrophys. J., 196, L129-L131, 1975.

Sun, W., Xu, S.-Y., and Akasofu, S.-I.: Mathematical separation of directly-driven and unloading components in the ionospheric equivalent currents during substorms, J. Geophys. Res., 103, 11695-11700, 1998.

Title, A.: in News detailed images of the sun, edited by: Kumar, M., EOS, 88, 3 April 2007.

Titov, V. S. and Demoulin, P.: Basic topology of twisted magnetic configurations in solar flares, Astron. Astrophys., 351, 707-720, 1999.

Tu, C.-Y., Zhou, C., Marsh, E., Xia, L.-D., Zhao, L., Wang, J.-X., and Wilhelm, K.: Solar wind origin in coronal funnels, Science, 308, 519-523, 2005.

Wang, H., Ewell Jr., M. W., Zirin, H., and Ai, G.: Vector magnetic field changes associated with X-class flares, Astrophys. J., 424, 436-445, 1994. 